\title{
5. Die Vereinbarkeit von Objektivität und Perspektivität
}

\subsection{Einleitung}

Es bleibt nun noch, die dritte Herangehensweise an den geschichtstheoretischen Widerstreit zwischen Standortgebundenheit und Objektivität zu betrachten: Historische Erkenntnis ohne Standort gibt es nicht. Entsprechend - und das sollten das zweite und das vierte Kapitel gezeigt haben - ist es weder eine Option, aus dieser Standortgebundenheit die Sinnlosigkeit jeglichen Objektivitätsanspruchs abzuleiten, noch ist es angängig, die Wissenschaftlichkeit der Geschichtsschreibung für unvereinbar mit der Standortgebundenheit historischer Darstellungen und Erkenntnisse zu erklären. ${ }^{262}$ Nicht nur hat die Begriffsexplikation des ersten Kapitels Korrespondenz

262 Vgl. Mommsen 1977: „Es ist zwar unbestritten, daß es auch in der Geschichtswissenschaft innerwissenschaftlichen Fortschritt in einem objektiven Sinne, nämlich der beständigen Akkumulation von objektiven Informationen über vergangene Wirklichkeit gibt, doch wird kaum jemand, der mit der Geschichte der Geschichtswissenschaft einigermaßen vertraut ist, die Ansicht vertreten, daß hierin das Wesen des historischen Erkenntnisfortschritts liege. Wenn [...] die Geschichte von jeder neuen Generation umgeschrieben werden muß - eine Proposition, die von keinem Historiker ernstlich bestritten werden dürfte -, so gewiß nicht nur deshalb, weil jeweils neue Tatsachen bekannt geworden sind, sondern vielmehr, weil sich die Gesichtspunkte gewandelt haben, unter denen vergangene Wirklichkeit als eine uns unmittelbar oder mittelbar angehende rekonstruiert bzw. erzählt wird.“ (Ebd., 444 f.) Vgl. Rüsen 2000, 63: „Only if the empirical evidence of the past is shaped according to the standpoints of those who communicate in the realm of historical consciousness and according to the value system which is derived from this standpoint does it acquire the quality of a plausible history; neutrality is the end of history." 
als Kern von Objektivität herausgestellt, auch haben die darauf folgenden Kapitel gezeigt, dass eine ästhetisierte oder radikal subjektivierte Geschichtsschreibung auf der einen Seite so wenig vertretbar ist wie eine radikal-objektivistische Geschichtsschreibung auf der anderen. Weder war die Option „Standortgebundenheit ohne Korrespondenz" noch die Option „positivistischer Objektivismus ohne erkenntniskonstitutives Erkenntnissubjekt" überzeugend. Elemente beider Seiten müssen zu einem Teil der Lösung gemacht werden. (Das gesehen zu haben, ist eine der wichtigen Einsichten des postmodernen Narrativismus.) Es muss gezeigt werden, wie es möglich sein kann, dass Geschichte der historischen Realität korrespondiert und dabei gleichzeitig die erkenntniskonstitutive Rolle des Standortes mit dieser Korrespondenz verträglich ist. ${ }^{263}$

Eine wichtige Voraussetzung für dieses Vorhaben ist insofern gegeben, als der naive, von Koselleck der vormodernen Geschichtstheorie attestierte Realismus und der auf ihn aufbauende Positivismus zumindest in der philosophischen Diskussion überholt ist. ${ }^{264}$ Das allgemeine erkenntnistheoretische Klima ist demnach günstig, um nun auch die Lösung des spezifisch geschichtstheoretischen Widerstreits erneut anzugehen. Möglich wird eine solche Lösung im Rahmen eines „scientific realism“, der den „God's Eye Point of View“265 des „metaphysical realism“266 als epistemologischen

263 Vor einigen Jahre hat H. Nagl-Docekal ihre Studie zu historischer Objektivität mit dem Schwebezustand enden lassen, in dem sich die Geschichtstheorie zwischen subjektivem Fundament der Historie einerseits und einem historistischen Objektivitätsideal andererseits befinde (dies. 1982, Kapitel IV: Das subjektive Fundament der Historie als Focus des Objektivitätsproblems). Über einen solchen Schlusspunkt soll hier hinausgegangen werden.

264 Vgl. Agazzi 2002, 47-52.

265 Putnams Gottesperspektive entspricht mutatis mutandis Nagels View from nowhere, d. h. einer epistemischen Position, von der aus die vollständige, unverzerrte Abbildung aller bestehenden Sachverhalte schlechthin möglich ist.

266 Putnam 1982, 75: Der metaphysische Realismus - so seine Terminologie für den naiven Realismus - sei eine Perspektive, ,wonach die Welt aus einer feststehenden Gesamtheit geistesunabhängiger Gegenstände besteht. Danach gibt es genau eine wahre und vollständige Beschreibung dessen, ,wie die Welt aussieht', und zur Wahrheit gehöre eine Art Korrespondenzbeziehung zwischen Wörtern bzw. Gedankenzeichen und äußeren Dingen und Mengen von Dingen. Diese Perspekti- 
Standard hinter sich gelassen hat, und der davon ausgeht, dass Erkenntnis nur, um hier Chladenius' Ausdruck zu verwenden, von einem „Sehepunct“ aus erfolgen kann, ohne dass dabei die Ansprüche auf Wahrheit oder Objektivität aufgegeben werden müssen.

Obwohl diese epistemologische Wende nicht allein in der philosophischen Diskussion gang und gäbe ist, sondern auch in der wissenschaftsund erkenntnisheoretischen Selbstreflexion der Geschichtswissenschaft etabliert ist - hierbei sind insbesondere Max Webers wissenschaftstheoretische Arbeiten von großer Bedeutung gewesen -, kann nicht davon gesprochen werden, eine unwidersprochene Selbstverständlichkeit zu vertreten. ${ }^{267}$ Zwar wird eine Vereinbarung von Objektivität mit Standortgebundenheit selten expressis verbis bestritten, aber nichtsdestotrotz tritt immer wieder ein mehr oder minder latenter Habitus zum Vorschein, aus dessen Äußerungen hervorgeht, dass erstens fundamentale positivistische, naiv-realistische Reflexe zumindest unterschwellig die Erkenntnistheorie der praktischen Geschichtsschreibung bestimmen. ${ }^{268}$ Hinzu kommt, dass zweitens auch wissenschaftstheoretische Argumentationen immer noch auf Restbestände eines solchen Positivismus zurückgreifen, was sich gerade an der argumentativen Auseinandersetzung mit der postmodernen Geschichtstheorie, aber auch in abgemilderter Form bei der Beurteilung narrativistischer Ansätze zeigt. Es kann also nicht eben davon gesprochen werden, offene Türen einzurennen, wenn man für eine perspektivische Geschichtstheorie argumentiert.

ve werde ich die externalistische nennen, denn ihr bevorzugter Gesichtspunkt ist das Auge Gottes." Der metaphysische Realismus ist, wenn man so will, die metaphysische Basis des Positivismus, auf der er ein ausdifferenziertes wissenschaftsund erkenntnistheoretisches Programm aufruhen lässt.

267 Vgl. Agazzi 2002, 47 f., wo eingewandt wird, dass kaum je ein Vertreter eines reinen „metaphysical realism“ gefunden werden könne, der sich wirklich jeden der genannten theoretischen Gehalte zu eigen machte.

268 Vgl. auch Kolmers Einschätzung, wonach sich ,zu dieser positivistischen , Theorie' [sc. dass aus den Quellen einzelne Fakten herausgelesen werden, die einfach aneinander gehängt werden können, um so die Vergangenheit, wie sie eigentlich gewesen ist, abbilden zu können] kaum noch jemand offen bekennen [mag]", weil „das Verdikt eines ,naiven Realismus“ im Raume steht“ (Kolmer 2008, 9 f. Herv. J. K.). 
Dieses Kapitel wird einige wichtige Grundelemente aufzeigen, die in einer Theorie enthalten sein sollten, die Standortgebundenheit und Objektivität vereinbaren möchte. Das positivistische Bild vom Erkenntnissubjekt als einem bloßen Durchlauf- und Wiedergabemedium vorhandener und auffindbarer Daten wurde zurückgewiesen, was nun dazu führt, dass an die Stelle dieses „Kübelbildes“ eine aktivische Auffassung von historischer Erkenntnisgewinnung tritt. Sie soll im folgenden als historischer Konstruktionismus bezeichnet werden, ohne damit viel mehr sagen zu wollen, als das, was auch im Konzept von Standortgebundenheit bereits enthalten ist: Erkenntnis ist zu einem (schwer festzulegenden) Grad auch vom Erkenntnissubjekt mitbestimmt. Auf welche Weise historische Erkenntnis von der Perspektive des Historikers mitbestimmt wird und wie dies möglich ist, ohne historische Objektivität aufgeben zu müssen und damit auf einen postmodernen Subjektivismus zu verfallen, soll in diesem Kapitel in Grundzügen gezeigt werden.

Um die Vereinbarkeit von historischer Objektivität mit historischer Standortgebundenheit zu plausibilisieren, wird im zweiten Abschnitt dieses Kapitels (5.2) vorgeschlagen, einen konstruktionistischen Weg zu einzuschlagen. Die ersten erläuternden Schritte zur konstruktionistischen These in diesem Abschnitt werden vor allem darauf abzielen, das Verhältnis von Perspektivismus und Konstruktionismus zu klären. Darauf muss (5.3) dem Einwand begegnet werden, Konstruktion impliziere oder bringe eine untragbare Willkür des Historikers mit sich. Zuletzt sollen in einem längeren Abschnitt zunächst einige theoretische Voraussetzungen dargestellt werden, welche die gesuchte Vereinbarung machbar werden lassen (5.4.1), worauf (5.4.2) einige Elemente der Perspektive aufgezählt und in ihrer Wirkungsweise wie auch in ihrer Interdependenz knapp dargestellt werden. 


\subsection{Perspektivismus und Konstruktionismus}

Eine schnelle Vereinbarung von Perspektivität mit Objektivität ließe sich erreichen, indem gleichsam beschwörend der Konstruktionismus angerufen wird und es dann mit der bloßen Erwähnung dieses Schlagwortes sein Bewenden hat. Immerhin kann der Konstruktionismus mit einer Vielfalt an Erscheinungsformen aufwarten, die es jedem ermöglichen würde, sich eine, seiner Ansicht nach geeignete Version oder einen Cocktail an geeigneten Versionen auszuwählen. Nicht selten werden ähnlich geartete Problemsituationen auch auf diese Weise gelöst. Bei solchen Gelegenheiten werden die bekannten, plakativen Hinweise auf dem Konstruktionismus endemische Konzeptionen und Thesen gegeben: die Unterbestimmtheit der Theorie durch die Fakten, die „Theoriegeladenheit“ der Beobachtungen, die „Seinsverbundenheit“" von Wissen, die Abhängigkeit der Untersuchung vom jeweiligen Erkenntnisinteresse u. v. m. ${ }^{269}$ Falsch ist diese ,Lösung' gewiss nicht, so wenig wie sie unangebracht ist. Schließlich kann sich der Konstruktionismus als legitime und wohletablierte erkenntnis- und wissenschaftstheoretische Doktrin betrachten, wie auch darüber hinaus der „Rahmen“, wie es immer wieder heißt, der meisten Arbeiten mit einer detaillierten Durchführung der konstruktionistischen Herangehensweise „gesprengt“" werden würde.

Auch die vorliegende Arbeit kann sich dieser Einschränkung nicht ganz entziehen, nur soll hier versucht werden, den konstruktionistischen Weg ein Stück weiter zu verfolgen, als dies meist geschieht. Es soll hier also nicht bei der schieren Beschwörung bleiben, wenn auch der Rahmen der vorliegenden Arbeit von einem vollständig durchdeklinierten historischen Konstruktionismus, das heißt von einer vollständigen konstruktionistischen Theorie historischen Wissens, „gesprengt“ würde. Wenigstens sollen im folgenden einige Grundzüge einer konstruktionistischen Theorie vorgelegt

269 Für einen Überblick über die verschiedenen Spielarten von „Konstruktismen“ (Hacking 2001), die sich auf dem theoretischen ,Markt" befinden, und deren jeweilige Kernaussagen vgl. Knorr-Cetina 1989, Hacking 2001 und, auf die Geschichtswissenschaft eingeschränkt, Goertz 2001, Kap. IV. 
werden.

Muss aber wirklich Zuflucht zum Konstruktionismus genommen werden, will man Perspektivität und Objektivität vereinbaren? Könnte nicht dafür argumentiert werden, dass ein nicht-konstruktionistischer Perspektivismus ausreichend wäre, um einerseits den korrespondenztheoretischen Kern der Objektivität zu bewahren, sich aber andererseits dennoch nicht in einen naiv-realistischen Positivismus schicken zu müssen? Um diese Fragen beantworten zu können, muss geklärt werden, wo die hauptsächlichen Differenzen zwischen Perspektivismus und Konstruktionismus zu finden sind. Beide werden zwar häufig in einem Atemzug genannt, müssen aber auseinander gehalten werden, weil der Konstruktionismus die ,stärkere', umfassendere theoretische Doktrin ist.

Die grundlegende Differenz zwischen nicht-konstruktionistischem Perspektivismus und dem Konstruktionismus besteht darin, dass der nichtkonstruktionistische Perspektivismus sich damit begnügen kann, darauf hinzuweisen, dass alle Erkenntnis notwendig partiell ist, weil es niemandem möglich ist, sämtliche Tatsachen, die auf einen Gegenstand zutreffen, in wahren Aussagen festzuhalten. Jede historische Darstellung muss sich auf gewisse Aspekte beschränken, die Gegenstand der Erkenntnis und der Darstellung werden sollen. Aus dieser Aspektivität folgt der Zwang zur Selektivität, das heißt, der Zwang, aus dem ,vorliegenden Material' (was auch immer damit im Einzelnen gemeint ist) jene Aussagen auszuwählen, die in die jeweilige historische Narration integriert werden müssen. Selektivität ist zwar auch ein ,Axiom' des konstruktionistischen ,Kanons', indes begnügt er sich bei der Bestimmung dessen, was Standortgebundenheit mit sich bringt, damit nicht.

Aus dem Selektivitätszwang ergibt sich eine weitere Frage. Was ist die Basis, aufgrund derer selegiert wird? Die inzwischen klassische Antwort auf diese Frage lautet: das Erkenntnisinteresse des Historikers. Auch hier liegt ein ,Axiom‘ vor, das vom Konstruktionismus geteilt wird: Nicht ausschließlich oder auch nur primär bestimmt das ,vorliegende Material', was Teil der Narration wird, sondern eben zum Beispiel das Erkenntnisinteresse des Historikers. Die Perspektive, von der aus wir auf die Vergangenheit bzw. auf die Quellen blicken, wird also in erster Linie davon bestimmen, 
was wir erfahren wollen.

Perspektivismus im nicht-konstruktionistischen Sinn bescheidet sich aber damit, das Phänomen narrativer Redeskription zu erklären, indem prima facie widersprüchliche Aussagen wie „der Berg liegt im Schatten“ und ,der Berg liegt in der Sonne“, als elliptische Aussagen betrachtet werden, die lediglich einer expliziten Formulierung des Standortes oder, mit Goodman gesprochen, des „Bezugssystems“ bedürfen. ${ }^{270}$ Die Widersprüchlichkeit der beiden Aussagen ließe sich auflösen, indem jeweils die Perspektiven, aus deren Blickwinkel sie geäußert werden, explizit in die Aussagen integriert werden: „der Berg liegt, von Norden aus betrachtet, im Schatten“ und ,der Berg wird, von Süden aus betrachtet, von der Sonne beschienen“. Genau in diesem Sinn, so könnte aus Sicht des Perspektivismus argumentiert werden, ließen sich verschiedene narrative Redeskriptionen desselben historischen Individuums und desselben Zeitabschnittes erklären und vereinbaren. Sie können als bloße Missverständnisse mittels Bezugskontextualisierung aus der Welt geschafft werden. Nimmt man als, im Grunde unwiderlegbare, Zusatzannahme hinzu, dass weitere Widersprüche faktisch vielleicht nicht aufgelöst werden können, weil die Quellenlage eine Auflösung nicht zulässt, obwohl sie prinzipiell möglich ist, erhält man einen beschränkten Perspektivismus, der in Übereinstimmung mit dem Positivismus zu dem Schluss kommen kann, dass sämtliche objektiven historischen Narrationen am ,Ende der Geschichte ${ }^{6}$ zu einer großen Narration vereinheitlicht werden können. Ebenso kann er, in Übereinstimmung mit dem naiv-realistischen Positivismus, an einer Abbildtheorie festhalten, wenn diese sich auch von einer schlichten, das heißt nicht-perspektivischen Abbildtheorie absetzt, indem er die Möglichkeit perspektivischer Selektivität anerkennt.

Wo genau beginnt nun der Konstruktionismus vom nichtkonstruktionistischen Perspektivismus abzuweichen, ${ }^{271}$ wenn beide die

270 Goodman 1984, 138 f.

271 Zwar könnte argumentiert werden, dass Selektivität eine Form der Konstruktion ist. Doch in der modernen Wissenschaftstheorie wird die Notwendigkeit zur Auswahl aus dem ,gegebenen Material' und die hauptsächliche Abhängigkeit der Kriterien dieser Auswahl vom Erkenntnissubjekt nicht angezweifelt. Derartige Versuche, einen nicht-konstruktionistischen Perspektivismus doch noch in das Boot 
Notwendigkeit der Selektivität und die Abhängigkeit der jeweiligen Auswahl vom Erkenntnisinteresse anerkennen? Es ist vor allem ein Gegensatz, durch die beiden voneinander geschieden werden: Der Konstruktionismus bestreitet, dass die Standortgebundenheit des Historikers nichts anderes impliziert, als die bloße Auswahl aus dem gegebenen Material. Der Standort des Historikers bestimmt (partiell) Gegenstand, Begriffe, Methoden der Untersuchung und darüber hinaus auch zu einem gut Teil das ,Material der Untersuchung. Standortgebundenheit wird also reichhaltiger verstanden als im nicht-konstruktionistischen Perspektivismus. Das bedeutet, dass historische Erkenntnis eine Funktion aus dem vorhandenen ,Material' und den erkenntnistheoretisch relevanten Voraussetzungen des Historikers ist. Welche das im einzelnen sind, ist immer wieder im Lauf der bisherigen Untersuchung zutage getreten. Sie werden aber im folgenden noch einmal gemeinsam vorgestellt werden. ${ }^{272}$

Wie kann taxonomisch das Verhältnis von Perspektivismus und Konstruktionismus bestimmt werden? Der Konstruktionismus kann als Spezies der Gattung „Perspektivismus im weiten, generischen Sinn“ betrachtet werden. Als Spezies dieser Gattung kann er auch als konstruktionistischer Perspektivismus bezeichnet und von einer anderen Spezies der Gattung „Perspektivismus“ unterschieden werden, die hier als nicht-konstruktionistischer Perspektivismus bezeichnet worden ist. Beide Spezies gehören zur selben Gattung, weil sie sich das fundamentale ,Axiom“ des Perspektivismus zu eigen machen: die Standortabhängigkeit (historischer) Erkenntnis. Sie müssen als Spezies jedoch getrennt werden, weil der konstruktionistische Perspektivismus Standortabhängigkeit vom gleichen Fundament aus, indes jedoch reichhaltiger interpretiert als der nicht-konstruktionistische Perspektivismus und beide darüber hinaus je andere erkenntnis- und wissenschaftstheoretische Schlussfolgerungen ziehen.

des Konstruktionismus zu zwingen, sind aus diesem Grund unwichtig.

272 Selbstverständlich werden nicht alle potentiellen Elemente in das Bild integriert werden können. 


\subsection{Ein Einwand gegen den Konstruktionismus}

Meist ruft allein die bloße Erwähnung des Begriffs „Konstruktion“ Widerstände und Einwände hervor. Mit diesen Einwänden wird darauf abgezielt, die Frage zu stellen, ob sich eine Abweichung von einer positivistischen Erkenntnistheorie tatsächlich mit historischer Objektivität vertragen kann. Schlimmer noch als die bislang verwendeten Begriffe „Standortgebundenheit" und „Perspektivität", deren passivische Konnotation immerhin noch einigen Raum für ein positivistisches Erkenntnismodell ließen, fordert der deutlicher aktivisch konnotierte Konstruktionsbegriff geradezu reflexartigen Widerstand heraus. Zugrunde liegt die Argumentation, Konstruktion bedeute aus eigener Willkür heraus hervorbringen. Wenn aber jeder nach eigener Willkür Geschichte konstruieren könne, dann könne wohl niemand mehr ernsthaft behaupten, die daraus resultierenden Narrationen korrespondierten mit der Vergangenheit. Objektivität könne folglich nicht mit Konstruktion vereinbar sein.

Hierbei handelt es sich jedoch um eine überzogene Vorstellung dessen, was Konstruktion bedeutet. Das Bild eines omnipotenten Baumeisters (historischen) Wissens führt in die Irre, weil es einen Konstrukteur voraussetzt, in dessen Willkür sämtliche Werkstoffe, Baupläne und Werkzeuge stehen. Es führt in die Irre, weil es nicht wirklich der Bedeutung von „Konstruktio$\mathrm{n}^{\text {“ }}$ angemessen ist: Dieses Argument gegen den Konstruktionismus verwendet den Konstruktionsbegriff begriffsaspektiv einseitig, das heißt, der begriffliche Gehalt von „Konstruktion“ gibt nicht das her, was das Argument unterstellt (vgl. 5.3.1). Hinzukommt, dass niemand die völlige Verfügung über seine Perspektive, von der aus er sein historisches Wissen konstruiert, besitzt, weil der Willkür des Historikers bei der Konstruktion des Geschichte faktische Grenzen gesetzt sind (vgl. 5.3.2). 


\subsubsection{Der begriffliche Gehalt von „Konstruktion“}

Im Kern des Einwandes gegen den Konstruktionismus steht die Frage, wie eng man „Konstruktion“ zu lesen geneigt ist. Der Einwand versteht unter „Konstruktion“ eine besonders intensive, allseitige Kontrolle durch den jeweiligen Konstrukteur oder Baumeister. Mit dieser einseitigen Lesart betont der Positivist die Kontrolle durch den Historiker und sieht darin eine Quelle epistemologischer Willkür oder Beliebigkeit.

„Konstruktion“ darf aber so eng nicht gelesen werden. „Konstruktion“ so eng aufzufassen, als sei sie mit Willkür und Beliebigkeit, frei waltender Imagination und Erdichtung identisch, bedeutet, einen von zwei begrifflichen Kernaspekten von „Konstruktion“ über Gebühr in den Vordergrund zu rücken. „Konstruktion“ führt nämlich zwei Konnotationen mit sich: zum einen die der Absichtlichkeit, zum anderen die des (kreativen) Wirkens mit bereits Vorhandenem. ${ }^{273}$

Wer also von einer Konstruktion von diesem oder jenem spricht, der meint damit einen (kreativen) Prozess, der willentlich abläuft und der zu einem Produkt führt, das nicht ex nihilo, sondern aus einem Reservoir vorhandener Techniken, Gegenstände, Werkzeuge etc. entstanden ist. Es kann also eine zweite, umfassende Explikation formuliert werden, die dem Konstruktionsbegriff angemessener ist: Die Konstruktion historischer Darstellungen ist ein Prozess, in dem sich gesteuerte mit ungesteuerten Elementen, willkürliche Akte mit restringierenden Bedingungen und individuelle Präsuppositionen mit plastischem, Material ' zu einer kontrollierten, nicht willkürlichen Konstruktion verbinden.

Der Vorwurf der Willkür rückt die Absichtlichkeit in den Vordergrund, vernachlässigt darüber die zweite Konnotation und übersteigert die Absichtlichkeit zu unkontrolliertem Phantasieren in luftleerem Raum. Demgegenüber muss der zweite Begriffsaspekt betont werden. Konstruktion bedeutet immer auch, aus bereits vorhandenen ,Bausteinen“ zu konstruieren. Damit sind konkret im Zusammenhang mit der Geschichtswissenschaft ,Fakten' wie auch Rahmennarrationen gemeint, darüber hinaus jedoch

273 Vgl. Becker 2002, 13 f. „Daß X von S konstruiert worden ist, impliziert nicht, daß $S$ anstelle von $X$ auch $Y$ hätte konstruieren können.“ (Ebd., 14) 
auch Methoden und Standards epistemischer Evaluation. „Konstruktion“ als rein subjektiv kontrollierte Willkür aufzufassen, bedeutet also einen begrifflichen Aspekt auf Kosten eines anderen, ebenso essentiellen begrifflichen Aspekts unstatthaft überzubetonen.

\subsubsection{Faktische Grenzen konstruktionistischer Willkür}

Im ersten Kapitel wurde als eine der derivativen Objektivitätsauffassungen des Komponentenmodells die konsensuale Objektivität eingeführt und diskutiert. Konsensuale Objektivität spielt, so wurde gezeigt, eine wichtige Rolle dabei, einer historischen Narration, einem Historiker und seinen Methoden Objektivität zuzusprechen. Über den Konsens innerhalb der Gemeinschaft wird inhaltlich festgelegt, was als objektive Erkenntnis gilt (was Objektivität im Vollsinn, i. e. als mit der Vergangenheit korrespondierend, nicht verbürgen kann), wer als unvoreingenommener Forscher und was als objektive Quelleninterpretation gilt. Der Konsens in der Gemeinschaft beschränkt damit faktisch die Willkür des Historikers bei der Konstruktion seiner Narrationen, weil sich kein Forscher einen dauerhaften, tiefgreifenden Verstoß gegen die Standards der jeweiligen Gemeinschaft leisten kann.

Dass die konsensuale Objektivität nicht unproblematische Konsequenzen mit sich bringt, etwa einen Konservatismus methodischen Standards oder thematischen Ausrichtungen gegenüber, trifft leider $\mathrm{zu}$, und leider trifft auch zu, dass damit sogar objektive Erkenntnisse verhindert werden können. Dieses Bedenken kann kaum restlos zerstreut werden. Allenfalls kann darauf gehofft werden, dass die schlimmsten Auswüchse dieses objektivitätsverhindernden Zugs der konsensualen Objektivität durch zum einen den interkollektiven Rationalitätsdruck zwischen den Forschergemeinschaften und zum anderen durch die Selbstverpflichtung der Wissenschaft zur Erlangung objektiver Erkenntnis, ihre wissenschaftliche Redlichkeit also, eingeschränkt werden können. Daneben muss aber auch dar- 
auf hingewiesen werden, dass mit dem Verweis auf die unerfreulichen, potentiellen Konsequenzen der konsensualen Objektivität kein Argument gegen eine konstruktionistische Vereinbarung von Standortgebundenheit und Objektivität gewonnen ist. Jede Geschichtstheorie wird sich damit auseinandersetzen müssen, dass die Mittel, mit deren Hilfe objektive Erkenntnis als solche erkannt werden soll, nicht frei von Mängeln sind, und zu diesen Mitteln wird für jede Geschichtstheorie auch die konsensuale Objektivität gehören müssen.

Konsensuale Objektivität ist aber nicht die einzige faktische Einschränkung, die sich die Willkür des Historikers gefallen lassen muss. Bevor überhaupt die scientific community ein Urteil über die Objektivität einer historischen Narration fällen kann, tritt eine Limitierung konstruktionistischer Willkür in Kraft, die bereits in der Perspektive des Historikers angelegt ist.

Seine Perspektive setzt sich aus gewissen Überzeugungen zusammen, die untereinander in einer Beziehung der Kohärenz stehen und die mittels bestimmter Begriffe ausgedrückt werden. Eine formale Bestimmung oder exakte Definition von Kohärenz zu geben, soll hier nicht versucht werden, es wird vielmehr der kolloquiale Gebrauch im Sinne von „Zusammenhang“, „Abhängigkeit“" und „Wechselwirkung“ vorausgesetzt. ${ }^{274}$ An dieser Stelle geht es nicht um den epistemologischen Kohärentismus als einer Rechtfertigungstheorie des Wissens. ${ }^{275}$ Es geht lediglich darum, einen Begriff dafür haben, dass die Überzeugungen des Historikers unmittelbar wie

274 Vgl. die ausweichende Antwort L. BonJours auf die Frage danach, was Kohärenz ist: „Intuitively, coherence is a matter of how well a body of beliefs ,hangs together': how well its component beliefs fit together, agree or dovetail with each other [...]. It is reasonably clear that this ,hanging together' depends on the various sorts of inferential, evidential, and explanatory relations which obtain among the various members of a system of beliefs [...].“ (BonJour 1985, 93)

275 Vgl. Quines berühmtes Diktum, wonach, ,unsere Aussagen über die Außenwelt nicht als einzelne Individuen sondern als ein Kollektiv vor das Tribunal der sinnlichen Erfahrung treten“. (Quine 1979, 45) Für eine modernere Spielart des Kohärentismus vgl. Elgin 2003, z. B. Seite 197 und auch 201: „Die Rechtfertigung einer Überzeugung ist keine Sache ihrer Ätiologie oder Phänomenologie, sondern hat allein damit zu tun, ob sie in ein haltbares System von Gedanken hineinpaßt.“" 
auch mittelbar aufeinander einwirken, so dass davon gesprochen werden kann, dass sie zu einem System zusammengeschlossen sind.

Dieses Überzeugungssystem lässt sich nicht vollständig über den Haufen werfen oder im Ganzen durch ein anderes ersetzen. Partiell kann das Überzeugungssystem verändert werden, aber immer nur mit Auswirkungen und unter dem (zumindest potentiellen) Veto anderer Überzeugungen. Nur vor dem Hintergrund seines Überzeugungssystems kann der Historiker neue Erfahrungen machen, Quellen analysieren, Tatsachen und Narrationen konstruieren. Jede neue Erfahrung, jede neue Narration muss kohärent zu seinem übrigen Überzeugungssystem sein. ${ }^{276}$ Sein Überzeugungssystem limitiert damit, wenn auch unter großen Spielräumen, seine konstruktive Willkür.

Gleiches gilt für den Begriffsrahmen, ${ }^{277}$ von dem der Historiker Gebrauch machen muss. Ein Begriffsrahmen ermöglicht ihm, bestimmte Din-

276 Als Minimalbedingung kohärenter Systeme wird in der Regel die Widerspruchsfreiheit des gesamten Überzeugungsystems bzw. der Subsysteme angegeben, das heißt, eine Widerspruchsfreiheit der im System enthaltenen Überzeugungen, neu gewonnener Überzeugungen und der Schlussfolgerungen aus ihnen.

277 Der Begriff des „Begriffsrahmens“ muss von dem des „Begriffsschemas“ unterschieden werden. Beide dienen der Identifikation und Klassifikation von Gegenständen. Sie sind in dem Sinn ,ontologisch' aufgeladen, als sie Gegenstände ,hervorbringen', weil die in ihnen enthaltenen Begriffe über ihre Verwendungsregeln bestimmen, was als Gegenstand gelten kann und welche Eigenschaften ihm über die ihn definierenden Eigenschaften hinaus zukommen. Sie legen, in den Worten Elgins, „,kategoriale Schemata“ fest. Begriffsschemata sind die Klasse sämtlicher dieser Anwendungsregeln zur Bestimmung von Gegenständen und ihren Eigenschaften, über die ein Erkenntnissubjekt oder eine Kultur oder gar die Menschheit verfügen. Sie sind in Sprachen ausdrückbar bzw. nur über sie ,verfügbar' (vgl. Abs. 4.2.3). Ein Begriffsrahmen, so zumindest in der Terminologie der vorliegenden Arbeit, ist eine Untereinheit des Begriffsschemas. Es kann von einem Begriffsrahmen des Alltags, der Wissenschaften generell oder aber der Geschichte, Physik, Psychologie usf. im Speziellen gesprochen werden. Damit soll nicht gesagt werden, dass hier eine strikte Trennung zwischen Begriffsrahmen vorliegt, sondern nur, dass bestimmte Begriffe (und damit Gegenstände und Eigenschaften) meist nur in bestimmten Kontexten relevant sind. In ihrer Rolle als Untereinheiten des Begriffsschemas sind sie aber untereinander verbunden und daher potentiell für alle Sprecher einer Sprache verfügbar. 
ge als Dinge von dieser und jener Art zu sehen, während er ihn für andere Dinge oder Eigenschaften ,blind' sein lässt. Ein Begriffsrahmen ist demnach ,ontologisch` aufgeladen. Auch dadurch erwächst seiner Willkür eine Limitierung, die er nicht einfach hinter sich lassen kann, weil er von irgendeinem Begriffsrahmen Gebrauch machen muss und dieser Begriffsrahmen qua Bestandteil des Begriffsschemas, das er verwenden muss, um sich überhaupt verständlich machen zu können, zum einen ansozialisiert ist, zum anderen aber auch einer permanenten sozialen Kontrolle unterliegt, die er nicht einfach umgehen kann.

Dabei entwickeln sich das Überzeugungssystem wie auch die verschiedenen Begriffsrahmen in steter Interdependenz. ${ }^{278}$ Neue Überzeugungen können neue Begriffe erfordern, neue Begriffe ermöglichen neue Überzeugungen, obsolete Begriffe lassen Rechtfertigungen obsolet werden etc. Gerade in der Geschichtswissenschaft dienen zum Beispiel die theoretischen Errungenschaften der Nachbardisziplinen als Denkanstöße und begriffliche Katalysatoren. Daneben entstehen neuartige Klassifikationen, das heißt, neue Begriffe werden eingeführt oder alte Begriffe umdefiniert. Einerseits besitzen wir eine gewisse Freiheit darin, ein neues „kategoriales Schema“ zu entwickeln, das ,einem Bereich eine Ordnung [aufzwingt], indem es einige Elemente als gleich, andere als ungleich klassifiziert", ${ }^{279}$ andererseits sind wir darauf festgelegt, dieses neue „kategoriale Schema“ in Einklang

278 Ein Wandel der Überzeugungen kann einen Wandel der Begriffe und umgekehrt mit sich bringen. Überzeugungen können nur mit dem vorhandenen Begriffsapparat ausgedrückt werden und Begriffe bedürfen der satzförmigen Erläuterung, Definition und Verwendung, um überhaupt erst einen Sinn zu haben. Beides ist Teil des evolutionären Wandels begrifflich-propositionaler Voraussetzungen neuer Wahrnehmung, Erfahrung und Erkenntnis. In Putnams Worten: „Durch den Gebrauch jedes Wortes - sei es ,gut ${ }^{\star}$ oder , bewußt' oder , rot' oder , magnetisch ${ }^{6}-$ wird man in eine Geschichte verstrickt, in eine Tradition des Beobachtens, des Verallgemeinerns, der Praxis und der Theorie. Man wird dadurch auch in das Unterfangen verstrickt, diese Tradition zu interpretieren und neuen Zusammenhängen anzupassen, sie zu erweitern und zu kritisieren. Man kann Traditionen auf verschiedene Weise interpretieren, doch man kann gar kein Wort anwenden, wenn man sich völlig außerhalb der Tradition placiert, der es angehört.“ (Putnam 1982, 268)

279 Elgin 2003, 199. 
mit unseren bislang festgehaltenen Überzeugungen und den mit ihnen zusammenhängenden kategorialen Schemata zu bringen (oder eben die überkommenen Systeme zu revidieren). ${ }^{280}$

Inwiefern ist dann aber der immerhin auch aktivisch konnotierte Konstruktionsbegriff noch angebracht, wenn neue Erfahrungen, neue Interpretationen und alternative Narrationen von vorhandenen Überzeugungssystemen und Begriffsrahmen limitiert werden? Sowohl sein Überzeugungssystem als auch seine Begriffsrahmen erwirbt sich der Historiker durch zuerst seine lebensweltliche und später seine wissenschaftliche Sozialisation. Hier scheint ihm wenig Raum für Beeinflussung oder gar Willkür zu bleiben.

Das ist soweit zwar korrekt, aber dennoch bleibt ihm Raum für eine permanente Modifikation sowohl seiner Begriffsrahmen als auch seines Überzeugungssystems. Modifizierbar bleibt sein Begriffsrahmen, wenn auch nur partiell und schrittweise. Der Historiker besitzt eine gewisse Verfügung über seine Begriffsrahmen, indem er gewisse Entscheidungen, im Idealfall aufgrund wissenschaftlich-rationaler Argumente, über die zu modifizierenden Bestandteile fällt. Auch sein Überzeugungssystem entwickelt sich weiter und dies nicht nur aufgrund von neuen Erfahrungen, die er mit den alten in einen kohärenten Zusammenhang bringt. Die vorhandenen Überzeugungen können durch neue Erfahrungen unter Druck kommen, weil - wie Quine zu bedenken gab - bei der Auflösung von Widersprüchen zwischen neuen und alten Überzeugungen fraglich ist, welche der widerstreitenden Überzeugungen geopfert werden muss. Nicht immer zieht die neue Überzeugung gegenüber der wohletablierten den Kürzeren.

Es sind aber nicht nur einzelne Überzeugungen, sondern ganze Überzeugungszusammenhänge, die untereinander in Beziehung stehen. Eine neue Überzeugung (eine neue Erfahrung) tritt unserem, mit Quine gesprochen, Netz aus Überzeugungen entgegen und wird entweder verworfen oder in dieses Netz integriert, wobei letzteres, im Fall des Widerspruchs der neuen

280 Elgin betont daneben auch, dass kategoriale Schemata (und die mit ihnen zusammenhängenden Überzeugungssysteme) auch Nützlichkeitserwägungen unterliegen: „Die nachgebesserte Klassifikation, die Wale zu Säugetieren zählt, ist vorzuziehen, so möchte ich behaupten, und zwar nicht nur deshalb, weil sie die Objekte, um die es geht, besser beschreibt, sondern weil sie mit den Interessen und Zielen der Biologie besser vereinbar ist.“ (Elgin 2003, 199) 
Überzeugung mit einigen Überzeugungen des Netzes, dazu führen kann, dass weitere etablierte Überzeugungen des Netzes fallengelassen werden müssen. Das Entscheidende an diesem Prozess ist, dass die Erfahrung, die am Rande des Netzes gemacht wird, zwar Einfluss auf die Zusammensetzung des Netzes besitzt, aber nicht die Zusammensetzung des Netzes restlos bestimmt. ${ }^{281}$ Das liegt zum einen daran, dass wir kein dem Netz externes Kriterium dafür haben, was wir im Fall des Widerspruchs verwerfen sollen und was nicht. Das liegt zum anderen aber auch daran, dass unsere Erfahrung immer im Licht der Überzeugungen und der in ihnen verwendeten Begriffe machen. Neue Erfahrung ist damit immer nur ein Stück weit vom bestehenden Netz entfernt, niemals etwas so radikal Neues, dass es mit dem Netz nicht in Widerspruch kommen kann. Neue Erfahrung wird immer im Rahmen des bewährten Netzes konzeptualisiert.

Dabei ist der Willkür bei der Entwicklung der Perspektive, i. e. des Überzeugungssystems und der diversen Begriffsrahmen, insofern wiederu$\mathrm{m}$ eine Grenze gesetzt, als sie sich ausschließlich im Zusammenhang mit anderen eigenen Überzeugungen und Präsuppositionen und den Überzeugungen anderer Menschen weiterentwickelt. Die Willkür des Historikers, sich zu konstruieren, was ihm beliebt, wird durch die Perspektive beschränkt, die sich zwar wandeln kann und die insofern auch wiederum seiner Willkür ausgeliefert zu sein scheint, die aber aufgrund ihrer nur partiellen, immer von einer neuen Perspektive abhängigen Wandelbarkeit (vgl. die Betrachtungen zur Objektivität als Unparteilichkeit: der Standort des Historikers kann sich immer nur von einem anderen Standort aus ändern, niemals von einem Nicht-Standort, einem View from nowhere aus) und dem beständigen Abgleich mit den Überzeugungen anderer Menschen auf einer höheren Ebene limitiert ist. Denn es kann grundsätzlich jede Voraussetzung hinterfragt werden kann, wenn auch nicht alle Voraussetzungen auf einmal. Welcher Standard, welche Methode, welches ,Faktum' oder welche Präsupposition auch immer - jeder dieser ,Knoten` kann zu seiner

281 Quine 1979, 47: „Doch das gesamte Feld ist so sehr durch seine Randbedingungen, durch die Erfahrung unterdeterminiert, daß wir eine breite Auswahl, welche Aussagen wir angesichts einer beliebigen individuellen dem System zuwiderlaufenden Erfahrung neu bewerten wollen." 
Zeit hinterfragt werden. Dabei mag es je nach seiner Lage im Netz leichter oder schwieriger sein, ihn als Voraussetzung zu hinterfragen. ${ }^{282}$ Jeder ,Knoten' ist, mit anderen Worten, einem beständigen Rationalitätsdruck unterworfen, der dem Historiker, neben seinem ohnehin vorhandenen kreativen Potential, genügend Raum lässt, seine erkenntniskonstitutive Perspektive jederzeit, wenn auch nur sukzessive zu modifizieren. So ist kein historischmaterialistischer Historiker dazu gezwungen, seine Geschichten als Episoden im Klassenkampf zu schildern. Er kann die Präsupposition, dass Geschichte im Grunde nichts anderes ist als eine Abfolge von Klassenkämpfen, hinterfragen und als doktrinär zurückweisen, so dass sein Blick frei wird für andere Rahmennarrationen oder spekulative Geschichtsphilosophien, die seine Erkenntnis leiten. Was er jedoch nicht tun kann, ist auf alle Geschichtsphilosophie verzichten (auch wenn einige Historiker dies vielleicht von sich behaupten mögen).

Ein Historiker ist also insofern nicht frei in seiner Willkür, als er auf irgendein ,Material' und irgendwelche Methoden zurückgreifen muss, während er dagegen insofern in seiner Konstrukteurswillkür unbeschränkt ist, als jede ,Gegebenheit' hinterfragt und zurückgewiesen werden kann, sofern gute Gründe vorhanden sind (und er nicht nachhaltig in Widerspruch zum fachdisziplinären Konsens gerät). Mittelalterliche Dinosaurier etwa kann der Historiker nur aus den Quellen herauslesen, wenn er bereit ist, seine paläontologischen und womöglich einen Teil seiner chronologischen Überzeugungen zu revidieren. Oder, um ein weniger gezwungenes Beispiel zu verwenden, eine mittelalterliche Monarchie als totalitären Verwaltungsstaat zu charakterisieren, bringt für ihn die Auflage mit, seine Überzeugungen hinsichtlich totalitärer Staaten, des Faschismus oder auch der administrativen Dichte des mittelalterlichen ,Staates' zu revidieren.

Angenommen aber, ein Historiker denkt vom Ergebnis her und sagt sich, diese These, die er (zum Beispiel) aus weltanschaulichen Gründen, unbedingt vertreten möchte, ist es ihm wert, sein gesamtes Überzeugungs-

282 In manchen Zusammenhängen mag es sogar völlig unnötig sein, wirklich alle Voraussetzungen als hinterfragbar zu betrachten. So kann es etwa für die Philosophie durchaus ein sinnvolles Streitthema sein, ob der Satz vom ausgeschlossenen Widerspruch hinterfragt werden kann. Für die Geschichtswissenschaft dürfte es reichlich sinnlos sein, diese Frage auch nur als potentiell relevant aufzuwerfen. 
system radikal zu revidieren. Selbstverständlich kann er dies tun, er ist aber, will er nicht gegen das Gebot der intellektuellen Redlichkeit, gegen das Unparteilichkeitsgebot und gegen die Gesetze der Logik verstoßen, gezwungen, einen ungleich schwierigeren und am Ende aussichtslosen Weg zu gehen, denn er wird gezwungen sein, immer zentralere Bestandteile des Überzeugungsnetzes zu revidieren, die mit anderen zentralen Überzeugungen zusammenhängen. Die Folge wird die sein, dass er sich, tritt er mit seinen Erkenntnissen an die Öffentlichkeit, in Widerspruch zum fachdisziplinären Konsens positioniert - und dies nicht nur in mehr oder minder eingeschränkten Bereichen, wie dies immer wieder vorkommt (und den wissenschaftlichen Fortschritt ausmacht), sondern in fundamentalen, grundstürzenden Überzeugungssegmenten des Netzes. Er wird darüber hinaus mit alltäglichen und auch fachfremden wissenschaftlichen Überzeugungen in Konflikt geraten. Er wird weiter und weiter in eine Isolation rutschen, welche die kommunikativen Voraussetzungen der Wissenschaft untergräbt. Es handelt sich um eine Art von Selbstentfernung aus seinem Fach.

Das gilt auch für die Kohärenz der Begriffsrahmen. Die Kohärenz, über die die verschiedenen begrifflichen Subsysteme untereinander verbunden sind, verhindert, dass Begriffe willkürlich verwendet werden. Kein Historiker kann sich willkürlich einen Staatsbegriff zurechtlegen, der es ihm erlaubt, den mittelalterlichen Staat als totalitären Überwachungsstaat zu konzeptualisieren. Der Staatsbegriff wird in einer Vielzahl begrifflicher Zusammenhänge verwendet, auf die eine derart radikale Umdeutung des Begriffs fatale Folgen hätte und die mit der entsprechenden Ablehnung durch die jeweiligen fachdisziplinären, vielleicht sogar alltäglichen Konsensgruppen sanktioniert werden würde. Die Weigerung, sich den begrifflichen Gepflogenheiten (und damit den jeweiligen Ontologien) anzupassen, würde im Extremfall dieselbe Isolation nach sich ziehen wie die starrsinnige Verletzung der Überzeugungskohärenz.

Ist der Historiker in seiner Willkür aber nicht in erster Linie durch die Quellen beschränkt? Weshalb sollten es der Konsens der Forscher und die Kohärenz neuer Erfahrungen mit seinen Überzeugungen und Begriffen (also seiner Perspektive) sein, die seine Willkür beschränken? Es sind doch immerhin die Spuren der Vergangenheit, die ihm erst Zugang zu ebendieser 
eröffnen und die seiner Willkür aus diesem Grund Restriktionen auferlegen.

Dieses Bedenken ist zwar korrekt, trägt jedoch nicht so weit, wie gemeinhin angenommen. Die Bearbeitung der Quellen, also ihre editorische Bearbeitung, ihre themen- und methodensensitive Relevanz und ihre anschließende interpretatorische Erschließung und Auswertung nach Maßgabe des jeweiligen Erkenntnisinteresses, ist alles andere als das häufig vorgestellte voraussetzungslose Aussieben oder Auffinden ,der Fakten'. Der Historiker ist zwar darauf festgelegt - sofern er Historiker bleiben möchte - aus Quellen gewonnene Fakten als solche zur Grundlage seiner Arbeit zu machen. Sein Gestaltungsspielraum erstreckt sich aber gerade bei der Konstruktion von Fakten sehr weit. Keine Quelle legt Art und Zahl der Fakten, die ein Historiker aus ihr gewinnt, fest. Zwar mögen Regestensammlungen den Eindruck erwecken, Historikern eine kanonische Abfolge von ,BasisFakten' zur Verfügung zu stellen, tatsächlich aber schreibt sich die kanonische Form gerade auch aus bestimmten Interessen und Präsuppositionen bestimmter Historiker her. So weit verbreitet diese Interessen und Präsuppositionen sein mögen, so wenig zwingend sind regestenartig zusammengestellte Fakten gegenüber den Fakten, die ein Historiker mit divergierenden Interessen und Präsuppositionen aus ihnen gewinnen würde.

Das bedeutet nicht, dass jeder Historiker Beliebiges aus beliebigen Quellen gewinnen kann. Quellen geben zwar nicht vor, was aus ihnen gewonnen werden kann, sie schränken aber sehr wohl ein, was aus ihnen gewonnen werden kann. In diesem Sinn meint Koselleck: „Strenggenommen kann uns eine Quelle nie sagen, was wir sagen sollen. Wohl aber hindert sie uns, Aussagen zu machen die wir nicht machen dürfen. Die Quellen haben ein Vetorecht. ${ }^{\text {“283 }}$ Koselleck erzählt damit aber nur die halbe Geschichte. Denn nur wenn wir bestimmte Standards besitzen, die uns erlauben oder verbieten, eine bestimmte Auffassung vom Inhalt einer Quelle zu haben, dann kann die Quelle ein Vetorecht besitzen. Diese Standards aber bringt nicht die Quelle bei, sondern bringen Methoden, Theorien und andere Präsuppositionen ins Spiel, die der Willkür des Historikers insofern entzogen sind, als er nicht willkürlich bestimmten, geltenden, und das heißt, in das

283 Koselleck 1979, 206. 
Überzeugungsnetz integrierten Überzeugungen einfachhin widersprechen kann.

Aber abgesehen von Bearbeitung der Quellen, sind die Quellen selbst nicht einfach etwas, das vom Historiker als Gegebenes betrachtet werden muss. Zwar wird er immer auf Quellen angewiesen sein, will er nicht zum historischen Romancier verkommen - soweit ist er auf ,Gegebenes' festgelegt und in seiner Willkür beschränkt -, nichtsdestotrotz besitzt er einen großen Gestaltungsraum: (1) Er kann eine bislang für einschlägig erachtete Quelle durch eine andere Quelle oder Quellengattung ergänzen. Die Ursachen des Ersten Weltkrieges etwa kann man ausschließlich in den außenpolitischen Akten der europäischen Mächte suchen oder man kann zusätzlich ein Psychogramm Wilhelms II. hinzuziehen. (2) Er kann eine bislang für einschlägig gehaltene Quelle auch zurückweisen. Vielleicht führen die offiziellen Aktenwerke der europäischen Mächte völlig in die Irre und einzig und allein die Psychologie Wilhelms II. plus die „Mentalität“ des „nervösen Zeitalters“'284 waren die Ursache des Ersten Weltkrieges. (3) Aber auch die Quellen selbst sind nicht sicher vor seiner Willkür. Die Akten des Auswärtigen Amtes etwa können von ihm als falsch im Sinne von „unglaubwürdig“ oder „falsch kompiliert“ oder „einseitig selegiert" etc. betrachtet werden. Auch die Quellen liefern daher nur relativ zu einer Perspektive eine Einschränkung der Willkür des Historikers. Aus sich selbst heraus und für sich allein sagen Quellen nichts und erst recht nicht, welche Tatsache aus ihnen herausgelesen werden können, um die Willkür es Historikers in ihre Schranken zu weisen. 


\subsection{3 Überleitung}

Es bleibt folglich, weist man den Willkürvorwurf zurück, noch die Option, sich für eine mehr oder minder paritätische Gewichtung beider eingangs erwähnten Begriffsaspekte zu entscheiden. Damit steht der Weg für eine detailliertere Darstellung des historischen Konstruktionismus offen. Konstruktion ist eine gleich gewichtete Kombination aus: persönlichem Erkenntnisinteresse, das dem Historiker mal existentiell (biographisch) widerfährt, mal aber auch willentlich von ihm gesetzt wird; ontologisch-metaphysischen Präsuppositionen, die meist, aber nicht unbedingt immer unhinterfragt bleiben, wohl aber im Laufe seiner Darstellungen zu Tage treten; theoretischen Neigungen, die ihm zunächst vielleicht aufgrund seiner fachdisziplinären Ausbildung unvermeidlich, später aber revisionsbedürftig erscheinen können; ,Fakten', die er ,wählt', wenn er sie für abgesichert erachtet, die er jedoch auch zurückweisen oder ignorieren kann, wenn er sie für uninteressant, falsch konstruiert oder gar für konträr zu (von ihm vorausgesetzten) elementaren metaphysischen Gesetzen oder theoretischen Modellen hält; selbst Quellen können mal unhinterfragtes Fundament der Darstellung sein, mal als nicht einschlägig für eine bestimmte Studie zurückgewiesen oder gar als Quelle für unbrauchbar erachtet werden; Fakten wird er zwar zugrunde legen, er ist aber in der Frage dessen, was ein Faktum für ihn ist, durch nichts in den Quellen festgelegt, vielmehr entscheidet er, welche Fakten die Quelle preisgibt. Die Liste an Gegebenheiten und Modifikationsspielräumen könnte noch verlängert werden. Anstatt aber auf diese enzyklopädische Weise fortzufahren, empfiehlt es sich, auf der Basis der bisherigen Erkenntnisse eine systematische Exposition einiger Grundzüge des konstruktionistischen Perspektivismus zu versuchen. 


\subsection{Grundzüge eines historischen Konstruktionismus}

Die Begriffsexplikation von „objektiv“/,Objektivität“ im ersten Kapitel hat ein Komponentenmodell erbracht, dessen integrativer Kern die korrespondenztheoretisch explizierte absolute Objektivität ist. Dieses Ergebnis ist wenig überraschend, werden doch der Geschichte immer wieder Funktionen wie die der Identitätsstiftung oder Politikberatung u. a. zugesprochen. Was also liegt näher, als der Geschichte einen Korrespondenzcharakter und dem Historiker die Fähigkeit, vergangene Tatsachen abzubilden, zuzuschreiben?

Weil sich aber dieser Common-sense-Positivismus bei genauerem Zusehen nicht uneingeschränkt halten lässt, hat sich als geschichtstheoretischer Widerpart die These von der notwendigen Standortgebundenheit oder Perspektivität historischer Erkenntnis ohne weiteres etablieren können, und auch sie kann eine kaum zu verleugnende Intuition für sich ins Feld führen. Die Geschichte wird immer wieder aufs Neue geschrieben, immer wieder anders interpretiert und bewertet, neue Fakten lassen sich aus bekannten Quellen herausarbeiten, alte Narrationen werden von neuen ersetzt oder ergänzt oder beide gemeinsam synthetisiert, längst Bekanntes wird zusammen mit völlig Unbekanntem immer wieder neu narrativ integriert. Dieses Phänomen der narrativen Redeskription ist aber mit der rudimentären, positivistischen Erkenntnis- und Wissenschaftstheorie des Common sense nicht leicht zu vereinbaren.

Von den drei im ersten Kapitel schematisch vorgestellten Lösungsversuchen dieses Widerstreits ist mittlerweile nur noch einer übrig geblieben. Den Widerstreit aus der Welt zu schaffen, indem man sich für eines und gegen das andere Extrem entscheidet, hat sich als unzulänglich erwiesen. Waren die vorangegangenen Kapitel in erster Linie dem Zweck gewidmet, diese ,extremistischen“ Lösungsansätze zu widerlegen, so sollten in ihnen aber auch, gewissermaßen kontrastiv, erste Bausteine für eine Lösung herausgearbeitet werden, die als ,third way“ zwischen den beiden Extremen und der einseitigen Verabschiedung entweder von Objektivität oder Standortgebundenheit hindurchführt. 
Diese (und einige der in diesem Kapitel erarbeiteten weitere) Bausteine sollen jetzt dazu benutzt werden, um den gesuchten ,third way“ in einer Vereinbarung von Objektivität und Standortgebundenheit in Grundzügen zu entwerfen. Diese Grundzüge sollen in zwei Schritten dargelegt werden. (1) Zunächst muss noch einmal die Korrespondenz, die zwischen Sachverhalt und Satzgehalt besteht, betrachtet werden und kurz umrissen werden, wie eine Einflussnahme des Standorts bei gleichzeitiger Korrespondenz der Darstellungen möglich sein kann (5.4.1). (2) Darauf werden einige zentrale Element des Standortes und ihre Wirkung auf die Geschichte beschrieben (5.4.2).

\subsubsection{Korrespondenz und Standortgebundenheit}

Der erste Schritt besteht darin, die Auffassung des epistemischen Zugangs zur Korrespondenz, d. h. zum Verhältnis zwischen Sachverhalt und Satzgehalt zu flexibilisieren. Die ontologische Unabhängigkeit des Sachverhalts von den mentalen Zuständen des Erkenntnissubjekts zusammen mit der Rede von Korrespondenz zwischen Tatsache und Satzgehalt legen ein zwar prima facie plausibles, nichtsdestotrotz aber an entscheidenden Stellen revisionsbedürftiges Bild nahe, wonach hier zwei von einander unabhängige Gegenstände existieren, von denen der eine, sc. die Tatsache, in der Welt $\mathrm{zu}$ finden ist, während der andere, sc. die Proposition, den kognitivbegrifflichen Gehalt, den diese Tatsache im Erkenntnissubjekt erhält, wiedergibt. Jeder Tatsache ist ein Satzgehalt zugeordnet, der ihr schon immer zugeordnet war und dies auch weiterhin bleiben wird und den es lediglich aufzufinden gilt. Im epistemischen Idealfall, das heißt, wenn das Erkenntnissubjekt nicht verzerrend eingreift, besteht eine Korrespondenz zwischen diesen beiden gegenständlich gedachten Relata, die betrachtet werden kann, indem das Erkenntnissubjekt beide Relata aus einer Mediatstellung heraus, die zwischen dem ,Reich“ der Tatsachen und dem der Satzgehalte angesiedelt ist, auf diese Übereinstimmung hin überprüft; die Tatsache und 
die Proposition werden gewissermaßen zur Hand genommen und auf Übereinstimmung hin untersucht.

Mit dieser naiv-realistischen Konzeption unseres epistemischen Zugangs zur (semantisch definierten) Korrespondenz lässt sich Standortgebundenheit kaum vereinbaren, weil das Phänomen der narrativen Redeskription, i. e. die immer neue, standortabhängige Beschreibung bereits untersuchter und dargestellter historischer Individuen, damit unvereinbar ist. Erkenntnisfortschritt, das heißt neue korrespondierende Aussagen und Narrationen, wäre nach dieser Konzeption nur möglich, wenn neue Quellen neue Tatsachen auffinden ließen. Was aber einmal nach methodisch korrekten Standards als objektive Narration erarbeitet worden ist, kann, sofern sie wirklich objektiv ist, nur durch weitere Tatsachen angereichert werden und auch dies nur aufgrund neuer Quellenfunde. Allenfalls könnten ,falsche Tatsachen' durch Quellenfunde oder eine verbesserte Methode ersetzt werden. Jeder Tatsache ist eine Proposition zugeordnet, die der Historiker zu finden und darzustellen hat. Gelingt ihm dies methodisch korrekt, werturteilsfrei und sprachlich neutral, dann ist über dieses historische Individuum, diesen Zeitabschnitt, diese Handlung, dieses Motiv etc. gesagt, was gesagt werden kann. Vielleicht würde noch zugestanden werden, dass wir tatsächlich niemals die Mediatstellung einnehmen können, von der aus wir die beiden Relata gegeneinander zu halten vermögen, trotzdem besteht das Ideal darin, eine solche Stellung einnehmen zu können, um von dort aus sämtliche Tatsachen mit den sie jeweils zugeordneten Propositionen ausmitteln zu können. Wobei dann die Aneinanderreihung dieser Tatsachen die wirklich objektive (Universal-)Geschichte darstellt, der möglicherweise noch in Form eines Appendix eine oder mehrere Interpretation(en) dieser Geschichte angehängt werden kann. In einem solchen Bild kann die Perspektive des Historikers allenfalls verzerrend wirken. Wozu sollte sie gut sein, wenn sowohl die Tatsache als auch die Proposition und dadurch beider Korrespondenz bereits feststehen, sie also nur noch (voraussetzungslos) aufgefunden und dargestellt werden müssen? In dieser Konzeption ist kein Platz für narrative Redeskription, weil sie nur dann möglich ist, wenn der Historiker nicht lediglich vorfindbare Tatsachen in Form von Propositionen auszudrücken hat, die er mit dem (methodisch korrekten) Auffinden 
der Tatsache gleich mit aufgefunden hat.

Nun wurde aber die narrative Redeskription als das zentrale Charakteristikum der Geschichte ausgemacht und die Ursache der narrativen Redeskription in der notwendigen Standortgebundenheit historischer Erkenntnis gesehen. Wenn aber narrative Redeskription bei gleichzeitiger Objektivität der Erkenntnis möglich sein soll, dann gelingt dies nur, wenn der Standort des Historikers einen verzerrungsfreien, also nicht korrespondenzverhindernden Einfluss auf die Darstellungen besitzt. Dies wiederum ist nur möglich, wenn die Vorstellung aufgegeben wird, die beiden unabhängigen, aber gleichwohl ewig einander zugeordneten Relata „Tatsache ${ }_{\mathrm{x}}$ “ und „Proposition ${ }_{\mathrm{x}}$ “ müssten nur aufgefunden und auf ihre Übereinstimmung überprüft werden, und schon stünde unveränderlich fest, was es zum jeweils in Betracht stehenden Gegenstand zu sagen gebe. An die Stelle dieser Konzeption soll eine flexibilisierte Auffassung treten, die hier skizziert werden muss, um den Blick auf die Funktionsweise des Standortes und seine Elemente frei zu bekommen.

Zwischen den Tatsachen auf der einen Seite und ihrer sprachlich-kognitiven Erfassung auf der anderen besteht ein strikt einseitiges Abhängigkeitsverhältnis. Tatsachen sind ontologisch von den sie erfassenden Satzgehalten (Propositionen) unabhängig. Umgekehrt dagegen besteht eine Abhängigkeit der Satzgehalte von den Tatsachen und zwar in dem Sinn, dass die Tatsachen die Inhalte der Satzgehalte mitbestimmen. Hierbei liegt die Betonung auf dem bloßen Mitbestimmungscharakter der Sachverhalte. Tatsachen allein bestimmen den Inhalt der Satzgehalte nicht ausschließlich. Eine entscheidende Rolle spielen dabei die Begriffe, mit denen die bestehenden Sachverhalte in sprachliche Form gebracht werden müssen.

Sachverhalte können nicht unbearbeitet und abbildhaft in den kognitiven Apparat des Beobachters einfließen. Propositionen sind logisch-semantische, probeweise Zusammenstellungen von Begriffen, die die Funktion haben, in der Beziehung der Korrespondenz zu ihren Gegenstücken (Referenten) in der Welt stehen und jenseits derer es nichts gibt, worauf wir zurückgreifen können, um Zugriff auf Sachverhalte zu haben ${ }^{285}$. Sprachlicher Zu-

285 Vgl. Patzig 1996; Lorenz 1997, 1998a und 1998b; Searle 1995, 205 f. Vgl. Wittgensteins Bemerkung von der probeweisen Zusammenstellung von Sachverhalten 
griff bedeutet, dass wir auf die aktuellen begrifflichen Möglichkeiten zur Darstellung von Sachverhalten festgelegt sind. Unser Begriffsschema (unsere Sprache) besitzt bestimmte begriffliche Möglichkeiten, Sachverhalte auszudrücken, andere dagegen - die nicht in der Ontologie des Begriffsschemas vorgesehen sind - können nicht ausgedrückt werden. Wir sind dazu in der Lage, Prädikate zu ,erfinden', also Extensionen festzulegen, die zuvor nicht vorhanden waren. Mit diesen neuen Prädikaten können neue Wahrheiten gefunden, also bekannte Gegenstände in neue Extensionen eingeordnet oder bekannte Gegenstände auf neuem Weg identifiziert oder gar völlig neue Gegenstände entdeckt werden. ${ }^{286}$

Das Begriffsschema teilt sich, wie bereits erläutert, in verschiedene Begriffsrahmen, die zwar nicht völlig voneinander abgeschlossen sind, die aber dennoch ein gewisses semantisches Feld abgrenzen. Sie stellen die jeweiligen Bereichsontologien zur Verfügung; zu denken ist dabei, an die verschiedenen Begriffsrahmen der Wissenschaften, aber auch des Alltags oder anderer spezieller Bereiche wie den der Astrologie o. ä. Die Erfassung von Sachverhalten und damit unsere Erkenntnis ist abhängig vom jeweiligen Begriffsrahmen. So ist zum Beispiel die Verwendung von Sortalen zur Identifikation von Gegenständen (,Individuen“) immer wieder hervorgeho-

in einer Aussage: „Im Satz wird gleichsam eine Sachlage probeweise zusammengestellt.“ (TLP 4.031); „Im Satz wird eine Welt probeweise zusammengestellt.“ (Tagebücher 1914-1918, Eintrag 29.9.14); „So stellt der Satz den Sachverhalt gleichsam auf eigene Faust dar.“ (ebd., 5.11.14).

286 Vgl. Elgin 2003, 197-200. „Die Extensionen der Prädikate können [..] vollkommen willkürlich sein. [...] Zweifellos lassen sich Wahrheiten erzählen bezogen auf eine Extension, die aus einer Muschelschale, Kleopatras Nase, der Mondscheinsonate und einem gebrochenen Antriebsriemen besteht. Würden wir ein Prädikat einführen, um eine solche Extension zu bezeichnen, wären wir zugleich in der Lage, solche Wahrheiten zu behaupten.“(Ebd., 198) Es mag an dieser Stelle erneut der Einwand hervorgebracht werden, dass damit ein Kulturrelativismus unvermeidlich sei. Wenn Satzgehalte von Begriffsrahmen (und damit von Begriffsschemata) abhängen, Begriffsrahmen aber kulturrelativ sind, dann müssen unsere Erkenntnisse, die ja in Satzgehalten ausgedrückt werden, ebenfalls kulturrelativ sein. Dass mit in diesem Sinn kulturrelativer Erkenntnis allerdings keine epistemologisch beunruhigende These einhergeht, ist bereits nachgewiesen worden vgl. Abs. 4.2.3. 
ben worden. ${ }^{287}$ Sortale ihrerseits sind jedoch Bestandteil kulturell bestimmter Begriffsrahmen. Allgemeiner formuliert: Ein Begriffsrahmen (oder mit Elgin: ein „kategoriales Schema“) wird kulturell entwickelt, modifiziert, aufgegeben usf. und bestimmt dabei aufgrund seiner klassifizierenden Funktion unsere Sicht auf die Welt, indem er festlegt, was es gibt und was nicht, was identisch mit dem einen Gegenstand und verschieden von einem anderen Gegenstand ist, was eine korrekte Gegenstandszusammenstellung ist und was als heterogene Gegenstände zu betrachten sind. ${ }^{288}$ Begriffsrahmen sind nicht Mittel zur Beschreibung verschiedener beobachterrelativer Welten, sondern Mittel verschiedener Beobachtungen ein und derselben Welt von beobachterrelativen Standorten aus. Dabei sind die Begriffsrahmen nicht starr und festgelegt, sondern unterliegen allerlei Wandlungen. Wer keinen Begriffsessentialismus (eine ,,magische Theorie der Bezugnahme" mit Putnam ${ }^{289}$ ) vertritt, das heißt, eine Position, wonach Begriffe exakt und genau bestimmten Begriffsumfängen und -referenten a priori und notwendig entsprechen, der muss davon ausgehen, dass Begriffsrahmen historische Produkte einer beständigen Wechselwirkung zwischen Menschen, Gruppen, Kulturen und Interaktionen derselben mit dem jeweils zur Verfügung stehenden Ausschnitt aus der Welt sind.

Wenn unsere Satzgehalte von historisch-kulturellen Begriffsrahmen mitbestimmt sind, dann ist das Geschehen in der Welt multiplen Beschreibungen zugänglich. Unsere Möglichkeit, die Welt mit diversen Begriffsrahmen zu ,überziehen', wandelt sich ständig, weil neue, alternative und vor allem: gleichberechtigte Klassifikationen zur Verfügung stehen. Das soll nicht bedeuten, dass es keinen Unterschied zwischen falschen und wahren/objektiven Aussagen gibt. Wahre Aussagen bleiben wahre Aussagen, sie sind aber durch weitere wahre Aussagen ad infinitum ergänzbar, kompilierbar oder auch weiter unterteilbar, je nach Perspektive auf einen Gegenstand und sei-

287 Vgl. Tugendhat 1976, 453 ff. „Das Charakteristische des Sortals ist, daß ein solches Prädikat [...] ein Kriterium des Identifizierens und Unterscheidens enthält; damit ist gemeint: durch das Prädikat ist vorgezeichnet, was alles, worauf gezeigt werden kann, zu dem einen Gegenstand - z. B. der einen Katze - gehört und was nicht." (Ebd., 453)

288 Vgl. Elgin 2003, 197-200.

289 Putnam 1982 passim. 
ne Eigenschaften.

Betrachtet man die bestehenden Sachverhalte, die sprachlich erfasst werden, als objektiv in dem Sinn, dass zwar ihr Bestehen unabhängig von Erkenntnissubjekten gegeben ist, die (kognitiv-sprachliche) Erfassung dieser bestehenden Sachverhalte jedoch subjektiv in dem Sinn ist, dass menschlichen Erkenntnissubjekten diese bestehenden Sachverhalte ausschließlich in Form von Propositionen gegeben sein kann, dann hat man das Fundament gewonnen, von dem aus begründet werden kann, wie historische Erkenntnis auf der einen Seite objektiv im Sinne der korrespondenztheoretischen Explikation und auf der anderen Seite dennoch standortgebunden sein kann.

Satzgehalte stellen die probeweise Zusammenstellung von Sachverhalten dar. Jenseits dieser Zusammenstellungen ist uns nichts gegeben. ${ }^{290}$ Erkenntnistheoretisch bedeutet dies, dass wir immer nur bestimmte Teile unserer Satzgehalte (in Form von Überzeugungen) vor der Folie anderer Satzgehalte (in Form von Überzeugungen) überprüfen können. Wir stellen also nicht willkürlich irgendwelche Satzgehalte zusammen, sondern sind an bestimmte Regeln, Normen, fundamentale Überzeugungen, Präsuppositionen etc. gebunden, die den Rahmen unserer konstruktionistischen Freiheit abstecken. Teil dieser Überzeugungen (und damit des Überzeugungssystems des Historikers) sind denn auch die Elemente des Standorts, die im folgenden Abschnitt eingehender vorgestellt werden sollen. Dabei sind die probeweisen Zusammenstellungen der Propositionen ebenso wie die Regeln, Methoden, ,Gesetze' wie auch die übrigen Überzeugungen des Überzeugungsnetzes von Begriffsrahmen abhängig. ${ }^{291}$

290 Als gegeben können nicht- oder vorbegriffliche Sinneswahrnehmungen angesehen werden. Aber abgesehen davon, dass Sinneswahrnehmungen für die Geschichte keine epistemologische Rolle spielen, weil sie auf kognitiv-begrifflichem Material, sc. den Quellen, basiert, können solche sensorisch gegebenen Data nur dann eine epistemische Funktion besitzen, wenn sie begrifflich-propositional eingekleidet werden.

291 Vgl. Putnam 1982, 81. Der Internalismus bestreite, „daß es Inputs [durch Erfahrung] gibt, die ihrerseits nicht durch unsere Begriffe geformt sind, durch das Vokabular, das wir zur Berichterstattung verwenden, und er bestreitet, daß es Inputs gibt, die nur eine einzige Beschreibung zulassen, die unabhängig ist von allen be- 
Wenn im Kern von Objektivität Korrespondenz stehen soll und Korrespondenz zwischen ontologisch von subjektiven Einstellungen unabhängigen Tatsachen einerseits und Propositionen andererseits bestehen soll, dann ist immer noch unklar, was unter Korrespondenz verstanden werden kann, wenn die Erfassung von Sachverhalten von Begriffsrahmen abhängig sein soll. Um überhaupt von Korrespondenz sprechen zu können, müssen die Strukturen der Tatsache sich in den Strukturen der Proposition wiederfinden lassen, andernfalls wäre von einer intersubjektiven Bedeutung von sprachlichen Ausdrücken nicht sinnvoll zu sprechen. Die Behauptung, dass $p$ muss als Behauptung, dass $p$ und nicht als Behauptung, dass $q$ oder Frage, $o b r$ o. a. vom Gegenüber verstanden und das heißt unter anderem, intersubjektiv übereinstimmend mit denselben (oder relevant ähnlichen) Strukturen in der Welt in Beziehung gesetzt werden (können).

Am nächstliegenden erfüllt der Isomorphismus, wie er etwa vom frühen Wittgenstein vertreten worden ist, diese Anforderung. Er hat aber seinen philosophischen Reiz inzwischen verloren. ${ }^{292}$ Mehr noch: Die strikte Identifizierung von Korrespondenz mit Isomorphismus ist gar nicht erst notwendig, weil eine ausreichend stabile Zuordnung von Begriffen und komplexeren, auf sie aufbauenden sprachlichen Ausdrücken zu den Gegenständen in der Welt genügt, um die genannte Minimalbedingung sprachlicher Verständigung zu erfüllen. Eine Zuordnungstheorie, die zwar feste Zuordnungen zwischen Begriffen (sprachlichen Termini) und Tatsachen (bzw. Gegenständen) vorsieht, aber auf eine Strukturübertragung von der Welt in den Satzgehalt hinein absieht, wäre hier denkbar. Aber auch eine solche Auffassung muss klären, wie sie dazu steht, dass Begriffe offensichtlich nicht ein für alle mal „magisch“ Gegenständen zugeordnet sind, sie wohl aber einer stabilen, wenn auch veränderbaren Zuordnungsfunktion bedarf.

Man kann dieses Problem lösen, indem eine Zuordnung von Begriffen grifflichen Entscheidungen".

292 Vgl. Newman 2002 und Putnam 1982, 84-88, für die frühe „Ähnlichkeitstheorie“ und ihre Schwierigkeiten, insbesondere die, dass Ähnlichkeit keine selbstevidente Beziehung ist, sondern immer einer supponierten, gesetzten oder vereinbarten Dimension bedarf, vor deren Hintergrund aus der unendlichen Vielzahl der Ähnlichkeitsbeziehungen zwischen zwei Gegenständen einige ausgesucht werden können (95 f.). 
und Gegenständen „intern“, das heißt, innerhalb von Begriffsrahmen (oder Begriffsschemata) vorgenommen wird. Die Sachverhalte, die sprachlich erfasst werden, hängen damit von den jeweiligen Begriffsrahmen ab, werden aber nichtsdestotrotz abgebildet, nur eben nicht so wie sie , an sich' oder von einer Gottesperspektive aus ,sind', sondern wie sie für uns aus der Perspektive eines Begriffsrahmens sich darstellen. Korrespondenz wird damit nicht mehr einem strikten Isomorphismus gleichgesetzt, der eine Ähnlichkeitsbeziehung zwischen zwei geistesunabhängigen, nichtsdestotrotz aber von uns als kognitiv finiten Wesen auf irgendeine mystische Weise inspizierbaren Gegenstandsbereichen stipuliert, sondern einer Zuordnung auf der (kulturell-variablen, wenn auch nicht willkürlichen) Basis von Begriffsrahmen.

Wenn nun also Sachverhalte insofern von unserem Begriffsrahmen abhängig sind, als uns nur diejenigen Sachverhalte zugänglich sind, die der jweilige Begriffsrahmen ,erlaubt ${ }^{6}$, kann Korrespondenz und damit Objektivität sehr einfach mit Perspektivität vereinbart werden. Korrespondenz besteht in der (uns zumindest intuitiv ohne theoretische Ausarbeitung zu Gebote stehenden) Übereinstimmung von Proposition und Tatsache. Der jeweilige Sachverhalt muss formuliert werden, was nur unter Rekurs auf den jeweils für relevant erachteten Begriffsrahmen geschehen kann. Dieser Begriffsrahmen stellt dabei nicht nur einfach Lautfolgen zur Verfügung, sondern auch sozial kontrollierte Weisen der Bezugnahme ${ }^{293}$ auf Gegenstände

293 Vgl. Wittgensteins Privatsprachenargument in den PU und Kripkes Rekonstruktion davon in Kripke 1982. Wittgensteins Privatsprachenargument zielt darauf ab, nachzuweisen, dass man einer Regel nicht ,privatim“ folgen kann, nicht einmal wenn es um die eigenen mentalen Vorgänge geht: „Und der Regel zu folgen glauben ist nicht: der Regel folgen. Und darum kann man nicht der Regel ,privatim“ folgen, weil sonst der Regel zu folgen glauben dasselbe wäre, wie der Regel folgen.“ (PU §202) Da es ohne Regeln keine sprachliche Bedeutung gibt, wir aber Regeln nicht privatim folgen können, bedürfen wir der Sprachgemeinschaft, wenn wir wissen wollen, ob wir wirklich einer Regel folgen, also tatsächlich einen Begriff richtig benutzen, sprich, ihn überhaupt ,besitzen'. (Vgl. Kripke 1982, 89, 95-99 und 107-109) Über einen Begriff verfügt man demnach, solange die einschlägige Sprachgemeinschaft ihre allzeit provisorische Anerkennung der individuellen Begriffskompetenz nicht revoziert: Wer zu oft einen Fehler bei einer Begriffsverwendung (von „Tisch“ etwa) macht, dem muss noch einmal erklärt 
aller Art (abstrakte wie reale Gegenstände, Zustände, Prozesse etc.). Dieses Bezugssystem wird ,intern', das heißt, innerhalb des Begriffsrahmens und unter Zuhilfenahme des Überzeugungssystems ${ }^{294}$ hergestellt, indem aufeinander aufgebaut und untereinander verwiesen wird. Wie diese interne Regelung austariert wird, muss eine Theorie des Spracherwerbs klären. Sicherlich kommt sie aber nicht durch einen (quasi-göttlichen oder pränatalen) Akt zustande, der, mit Putnam gesprochen, Gegenstände „magisch“ mit ewiggültigen Bedeutungen in Beziehung setzt. Erlauben unsere Begriffsrahmen bestimmte Bezugnahmen auf Gegenstände und erlauben sie die Anwendung von Begriffen auf Eigenschaften, das heißt, können Begriffe im Satz die Funktion von singulären und generellen Termini übernehmen, dann liegt, gelingt die Anwendung des generellen Terminus auf den singulären Terminus nach Maßgabe der im Begriffsrahmen festgelegten und sozial kontrollierten Regeln der Bezugnahme, Korrespondenz vor.

Diese interne Regelung von sprachlicher Bedeutung vorausgesetzt, kann deutlich gemacht werden, wie narrative Redeskription bei gleichzeitiger Korrespondenz möglich ist. Wenn (i) Sachverhalte nur sprachlich zugänglich sind, und wenn (ii) sprachliche Abhängigkeit sich als Abhängigkeit von einem Begriffsrahmen (und den damit verbundenen Regeln der Begriffsverwendung) auffassen lässt, und darüber hinaus (iii) Begriffsrahmen dem Wandel unterliegen, dann ist narrative Redeskription möglich, weil die Korrespondenz zwischen Tatsachen und Propositionen nicht mehr ein für alle mal feststeht, sondern ihrerseits eine evolutionäre Dimension besitzt, welche die Voraussetzung dafür ist, dass dieselben historischen Vorgänge immer wieder aufs Neue narrativ dargestellt werden. Neue Begriffe und alte Begriffe mit neuen Anwendungsregeln bringen die Möglichkeit mit sich, neue Wahrheiten auch an Gegenständen entdecken zu können, die uns bereits geläufig sind. Dabei handelt es sich um kein ausschließlich historisches Phänomen. Eine neue Kosmologie bringt neue potentielle Wahrheiten über dieselben Planeten zum Vorschein, die schon seit langem beob-

werden - der muss, mit Wittgenstein gesprochen, noch einmal ,abgerichtet“" werden -, welche Bedeutung der Begriff eigentlich besitzt.

294 Das ist wichtig, weil bestimmte Bezugnahmen aufgrund von Überzeugungen gegenstandslos werden können; das „Phlogiston“ etwa. 
achtet werden; neue zoologische Klassifikationen bringen neue Wahrheiten über bekannte Spezies mit sich; die soziologische Konzeptualisierung von sozialen Verhältnissen bringt für die darauf folgende Forschung die Möglichkeit mit sich, über bereits betrachtete Kollektive neue Wahrheiten zu entdecken, die bislang nicht entdeckbar waren, weil der begriffliche Rahmen nicht gegeben war. Diese neuen Wahrheiten hätten im alten Begriffsrahmen selbst mit genauester Beobachtung nicht entdeckt werden können, mit einem neuen Begriffsrahmen dagegen können sie sich dem Forscher geradezu aufdrängen. Unsere Möglichkeiten, immer neue objektive Darstellungen von längst Erforschtem zu geben und Altes neben Neues zu stellen (und natürlich auch immer wieder alte ,Wahrheiten' als falsch zu erweisen) endet demnach erst mit dem Ende des Perspektivenwandels, mit der Lösung aller „Kulturprobleme“, kurz, mit dem Ende der Geschichte.

Einem solchen Vorschlag wird gewiss der Einwand entgegengebracht, dass damit Objektivität „relativ“" geworden sei. Wenn damit gemeint sein sollte, dass die Korrespondenz unserer Propositionen mit den Tatsachen von unseren Begriffsrahmen abhängig ist (weil nur die Propositionen formuliert werden können, für die wir die begrifflichen Mittel besitzen), dann muss dem zugestimmt werden. Wenn damit gemeint sein sollte, dass damit alle Erkenntnis subjektiv und alle Wahrheiten Scheinwahrheiten sind, weil es nur eine Wahrheit geben kann - ein ,Missverständnis', das zumindest billigend in Kauf genommen wird -, dann muss erneut darauf verwiesen werden, dass ein solcher Relativismus ausgeschlossen ist. Solange wir unsere Begriffsrahmen und die Aussagen, die auf ihnen basieren, ineinander übersetzen können, besteht immer die Möglichkeit, Aussagen zu überprüfen oder sich diskursiv über ihren Objektivitätsgrad rational auseinanderzusetzen. Darüber hinaus garantiert die sprachliche Sozialisation die gesuchte Stabilität der Zuordnung zwischen dem Begriffsschema (und seinem sprachlichen Ausdruck) und den Gegenständen und Sachverhalten in der Welt.

Eine letzte Erläuterung ist an dieser Stelle darüber hinaus notwendig: Wenn hier davon gesprochen wird, dass Korrespondenz abhängig von Begriffsrahmen ist, dann ist damit eine epistemologische Konzeption vorgetragen. Damit ist nicht gemeint, dass die korrespondenztheoretische Expli- 
kation des Wahrheitsbegriffs auf einmal um einen Zeit- oder Begriffsrahmenindex ergänzt werden müsste. Satzgehalte, die wahr sind, sind wahr und damit sind die Sachverhalte, die von ihnen ausgedrückt werden bestehende Sachverhalte, i. e. Tatsachen. ${ }^{295}$ Dieses zeitlose Verhältnis besteht eins zu eins; dies ist eine logisch-semantische Explikation des Wahrheitsbegriffs. Was dagegen den sozio-kulturellen Wandlungen unterworfen ist, ist unsere Fähigkeit, Propositionen zu formulieren und ihren Wahrheitsgehalt zu überprüfen. Erstere Fähigkeit modifiziert nicht den Wahrheitsgehalt einer Aussage, noch das eben ausgeführte logisch-semantische Verhältnis, das den Wahrheitsbegriff korrespondenztheoretisch expliziert. Diese Fähigkeit hat lediglich die oben skizzierte Relevanz, dass mit dem Lauf des sozio-kulturellen Begriffswandels und des (wissenschaftlichen) Erkenntnisfortschritts immer neue Begriffe zu Verfügung stehen, mit deren Hilfe wir Sachverhalte in neue, bislang nicht formulierbare Propositionen bringen können. Damit sind uns Sachverhalte und mit ihnen wiederum Wahrheiten zugänglich, die insofern neu sind, als sie uns bislang nicht zugänglich waren; ihren Wahrheitswert ,wahr“ besitzen sie selbstverständlich zeitlos. Die Korrespondenz, die in diesem zeitlosen Wahrheitswert zum Ausdruck kommt, ist uns als endlichen Erkenntnissubjekten wiederum nur zugänglich, indem wir kognitiv zur Welt stehen.

Dieser kognitive Zugang zur Welt unterliegt seinerseits wieder gewissen sozio-kulturellen Wandlungen und zwar in dem Sinn, dass wir bestimmte Methoden, den Wahrheitsgehalt einer Proposition zu überprüfen und zu rechtfertigen, für besser oder schlechter ansehen. Auch diese Wandlungsfähigkeit insbesondere der wissenschaftlichen Methode bringt, absichtlich lax formuliert, ,neue Tatsachen' nur insofern in die Welt, als Propositionen auf andere Weise auf ihren zeitlosen Wahrheitsgehalt überprüft werden und entsprechend ihren Wahrheitswert, ändern' (oder eben auch nicht). Das heißt, dass sich der Wahrheitswert einer Proposition für uns ändert, aber

295 Das ist die ontologische Seite der korrespondenztheoretischen Wahrheitsbegriffs. Und das ist natürlich auch der Witz an unserer Suche nach Wahrheit. Wahrheit wird ja in der Regel nicht gesucht, um Wahrheit zu besitzen, sondern um zu wissen, wie die Welt beschaffen ist, d. h. wer eine (gesichert) wahre Aussage in Händen hält, ist damit ontologisch aufgeklärt. Die Welt ist so, wie die wahre Aussage sagt. 
nicht in einem logisch-semantischen Sinn, sondern in einem epistemischen Sinn. Eine Gottesperspektive würde diesem Wandel nicht unterliegen.

Wenn also der Standort des Historikers Einfluss auf den Wahrheitswert einer Aussage oder, wie es für die Geschichtswissenschaft besser angesetzt ist, einer Narration, also auf ihre Korrespondenz oder Nicht-Korrespondenz und damit letztlich ihre Objektivität hat, dann ist damit nicht eine Wandlung des zeitlosen logisch-semantischen Verhältnisses zwischen Aussagen und Ausgesagtem, sondern unseres Zugangs zu diesem Verhältnis gemeint. Für die Gottesperspektive gibt es diesen Wandel nicht, weil ein Wesen, das die (für uns Menschen), mit Kutschera zu reden, „falsche Objektivität" tatsächlich besitzt, über restlos alle zeitlosen Wahrheiten verfügt, die sich nicht-göttliche Wesen immer nur partiell und immer nur unter der epistemologischen Restriktion einer dräuenden Revision und mit Hilfe kontinuierlich modifizierbarer wie modifizierungsbedürftiger Begriffsrahmen erarbeiten müssen.

Damit geht die Bedeutung des sozio-kulturell konstituierten Standorts des Historikers über die Funktion hinaus, die - nach dem nicht-konstruktionistischen Perspektivismus - nur darin besteht, Relevanzkriterien, also gleichsam den Filter, für bestimmte Tatsachen in Form eines Erkenntnisinteresses zu liefern. Die Abhängigkeit historischer Objektivität ist in diesem Zusammenhang vom Wandel der Begriffsrahmen (und damit: vom Ontologiewandel) und ebenfalls wandelbaren Standards wissenschaftlicher Rechtfertigung von Korrespondenzansprüchen tiefer im Standort des Erkenntnissubjekts verankert, als es das bloße Beschaffen eines Erkenntnisinteresses vorsieht. 


\subsubsection{Die Elemente des Standortes und ihr Einfluss auf his torische Erkenntnis}

Der Standort des Historikers setzt sich aus bestimmten Überzeugungen zusammen, die ihren Ausdruck in den verschiedensten Begriffsrahmen finden. Begriffsrahmen sind stabile, wenngleich wandelbare interne Zuordnungen von Begriffen zu Gegenständen. Sie geben die Möglichkeiten an die Hand, Gegenstände und ihre Eigenschaften zu identifizieren. Sie sind daher ,ontologisch aufgeladen", das heißt, was der Begriffsrahmen zu einem Zeitpunkt $t_{1}$ nicht als Gegenstand zu identifizieren erlaubt oder als Eigenschaft kennt, das kann nicht als Gegenstand oder dessen Eigenschaft kognitiv-sprachlich erfasst werden. $\mathrm{Zu}$ einem späteren Zeitpunkt $t_{2}$ kann der Wandel der Begriffsrahmen (und der Überzeugungen des Erkenntnissubjekts) eine neue Ontologie, neue Gegenstände und Eigenschaften identifizieren lassen. Der Zugang zu Sachverhalten findet ausschließlich über diese Begriffsrahmen statt. Sachverhalte werden probeweise in Propositionen zusammengestellt und je nach Übereinstimmung mit den übrigen Überzeugungen auf ihre Korrespondenz hin eingeschätzt.

Auf diese Weise ist es möglich, Korrespondenz mit Standortgebundenheit in Einklang zu bringen, weil so erklärt werden kann, wie der Standort immer neue narrative Redeskriptionen hervorbringen kann, ohne damit gleichzeitig alte Narrationen als falsch verwerfen zu müssen. Die ihrerseits historische Wandlung des Überzeugungssystems (i. e. des Standortes) bringt die Wandlung der Begriffsrahmen mit sich. Dadurch wiederum werden bestimmte neue Gegenstände identifizierbar und neue Eigenschaften erkennbar. Zum Teil werden alte Gegenstände und ihre Eigenschaften als ontologisch überholt betrachtet werden müssen, zum Teil wird die alte Ontologie angereichert werden, folglich gehen Ergänzungen mit Ersetzungen einher.

Freilich handelt es sich bei verschiedenen Begriffsrahmen, mit denen die Möglichkeit einhergeht, ,neue' Wahrheiten zu entdecken und zu formulie- 
ren, um den Extremfall historischer Perspektivität. Perspektivische Effekte kommen selbstverständlich auch dann zustande, wenn Historiker sich einen (oder mehrere) Begriffsrahmen teilen, sprich, von der Nützlichkeit eines bestimmten Begriffsrahmens (etwa dem der Psychologie oder dem der Ökonomie) für ihre konkrete Fragestellung überzeugt sind, aber in ihrem Erkenntnisinteresse divergieren oder eine andere Quellenselektion vornehmen u. a. In einem Kontinuum historischer Perspektivitätsbedingungen stellen die Divergenzen durch differierende Begriffsrahmen den Pol maximaler Perspektivendivergenz dar, während etwa divergierende Erkenntnisinteressen ceteris paribus keinen derart fundamentalen Perspektivenwandel hervorbringen. Zwischen diesen beiden Polen liegen die weiteren Bedingungen historischer Perspektivität wie unterschiedliche Methodologie, theoretische Vorannahmen oder auch Quellenselektion und -interpretation.

Diese Zusammenfassung der Ergebnisse des letzten Abschnitts sollen als Hintergrund und theoretische Voraussetzung für eine Skizze der Wirkungsweise der Perspektive des Historikers dienen. Zu diesem Zweck werden was wiederum nur in Grundzügen geschehen kann - diverse Elemente des bislang lediglich abstrakt als Überzeugungssystem charakterisierten Standortes in ihrer Wirkungsweise auf die historische Erkenntnisgewinnung dargestellt werden. Dabei soll sich auch zeigen, dass die Bezeichnung ,historischer Konstruktionismus" für diese Konzeption insofern angemessen ist, als der Historiker (durch seine Perspektive) durchaus (und im Gegensatz zum eventuell entstandenen Eindruck) aktiv Anteil an der Erkenntnisgewinnung hat und kein bloßes Durchlaufmedium ist.

Dies soll auf vier Ebenen gezeigt werden: (1) Die Konstruktion von historischen Individuen als Gegenstände der Geschichte und als Protagonisten historischer Narrationen (5.4.2.1); (2) die Ebene der Narrationsbildung, die zugleich die ,Tatsachenkonstruktion' mitumfasst (5.4.2.2); (3) die Ebene der theoretischen Vorannahmen, der Werte, des Erkenntnisinteresses und der Metaerzählungen (5.4.2.3); (4) die Ebene der Metareflexion auf die verschiedensten Elemente des Standortes, wie etwa Methoden, Erkenntnisinteresse, Begrifflichkeiten etc. (5.4.2.4). 


\subsubsection{Die Konstruktion historischer Individuen}

Der Standort des Historikers wirkt zunächst über die Konstruktion historischer Individuen im Weberschen Sinn. ${ }^{296}$ Der Historiker segmentiert durch die Konstruktion historischer Individuen den unendlichen Fluss vergangener Ereignisse. In erster Linie erreicht er die Segmentierung, indem er durch die Konstruktion historischer Individuen festlegt, was Gegenstand der Untersuchung werden kann, was also ein historischer Gegenstand ist er konstruiert also das, was der Gegenstand der Narration, sprich, ihr ,Protagonist" ist.

Weber stellt sich diesen Vorgang folgendermaßen vor: Historische Individuen sind die Antwort auf die Frage nach der Möglichkeit, den hiatus irrationalis zu überwinden, der zwischen den Ereignissen und der begrifflich-kognitiven Erfassung der Realität klafft. ${ }^{297}$ Die Naturwissenschaften überbrücken den hiatus irrationalis, indem sie sich auf quantifizierbare Begriffe und Gesetze spezialisieren, die auf möglichst viele Gegenstände zutreffen sollen. Die Naturwissenschaften zielen dabei auf die (möglichst restlose) Subsumption dieser Gegenstände unter Gesetze oder GesetzmäBigkeiten ab. ${ }^{298}$ Die Kulturwissenschaften hingegen seien auf die konkrete Realität eines Gegenstandes konzentriert, das heißt, sie sind an den ,individual and qualitative properties [of reality] as such“6299 interessiert. Sie sind „Wirklichkeitswissenschaften“, weil sie ihr Augenmerk auf diese konkrete, qualitative Beschaffenheit singulärer Gegenstände und deren kausa-

296 Auf Webers Theorie kulturwissenschaftlicher Gegenstandskonstruktion soll hier zurückgegriffen werden, weil sie meiner Kenntnis nach die elaborierteste und avancierteste Theorie ist, die sich mit der perspektivischen Erschaffung von historischen Individuen auseinandersetzt; und in diesem Zusammenhang ist gerade Webers Betonung von einerseits subjektiven Voraussetzungen bei gleichzeitiger kulturwissenschaftlicher Objektivität andererseits von besonderem Reiz für die vorliegende Arbeit.

297 Zum hiatus irrationalis vgl. 4.2.1 und dort insb. Anm. 204.

298 OA, $171 \mathrm{f}$.

299 Oakes 1988, 22 f. 
ler Genese richten. ${ }^{300}$

Wie kann aber aus der unendlichen Mannigfaltigkeit der Realität ein Phänomen ausgewählt werden, wenn nicht einmal die Möglichkeit besteht, mit der Vielzahl der Phänomene durch Subsumption unter ,generelle Begriffe" fertig zu werden, wie dies die Naturwissenschaften anstreben und partiell auch verwirklichen können? Anders formuliert: Was lässt eine Vielzahl von Phänomenen zu einem Gegenstand kulturwissenschaftlicher Untersuchung werden? Die Antwort gibt Weber bereits an früher Stelle in der WL: „In allen diesen Fällen [sc. kausal bedingter, historisch folgenreicher, psychisch wie nicht-psychisch bedingter Ereignisse und Prozesse] ist [..] der Sinn, den wir den Erscheinungen beilegen, d. h. die Beziehung auf ,Werte', die wir vollziehen, dasjenige, was der ,Ableitung' aus den ,Elementen' als prinzipiell heterogenes und disparates Moment die Pfade kreuzt. Diese ,unsere` Beziehung ,psychischer' Hergänge auf Werte, [...] vollzieht eben die ,schöpferische Synthese'.، ‘301

Wie geht diese Auswahl und Synthese vor sich? Für Weber besteht die kategoriale Differenz der Kulturwissenschaften gegenüber den Naturwissenschaften einzig im ,deutenden Verstehen“ - alle anderen Optionen, ein spezifisches Merkmal der Kulturwissenschaften vorzulegen, scheitern nach Webers Ansicht. Deutendes Verstehen kann dreierlei bedeuten: (1) „Anregung zu einer bestimmten gefühlsmäßigen Stellungnahme“ - dann sei sie „Zumutung zum Vollzug einer Wertung“; (2) „Zumutung eines Urteils im Sinn der Bejahung eines realen Zusammenhanges als eines gültig ,verstandenen““ - „dann ist sie [...] kausal erkennende ,Deutung““; (3) zwischen beiden liegt die, später so genannte, „Wertinterpretation“. ${ }^{302}$ Letztere ist

300 Roscher-Aufsatz, 113; vgl. auch OA, $171 \mathrm{ff}$.

301 Roscher-Aufsatz, 54. Vgl. OA, 175: „Die Bedeutung der Gestaltung einer Kulturerscheinung und der Grund dieser Bedeutung kann aber aus keinem noch so vollkommenen System von Gesetzesbegriffen entnommen, begründet und verständlich gemacht werden, denn sie setzt die Beziehung der Kulturerscheinungen auf Wertideen voraus.“ „Was aber Bedeutung hat, das ist natürlich durch keine ,voraussetzungslose' Untersuchung des empirisch Gegebenen zu erschließen, sondern seine Feststellung ist Voraussetzung dafür, daß etwas Gegenstand der Untersuchung wird.“(OA, 175 f.)

302 Roscher-Aufsatz, 89. 
weder eine Wertung wie in Fall (1) noch ist sie eine kausale Kategorie wie im Fall (2). Die Wertinterpretation, die in den späteren Kritischen Studien auf dem Gebiet der kulturwissenschaftlichen Logik von zentraler Bedeutung sein wird, bereitet lediglich das deutende Verstehen vor, indem sie „mögliche Wertbeziehungen eines Objekts“ herausarbeitet. Die Wertinterpretation ist es, die das historische Individuum vorbereitet, in dem sich dann die „Synthese“ vollzieht, welche die Voraussetzung eines jeden Deutens bzw. Verstehens ist, weil durch sie erst die Gegenstände konstituiert werden, die gedeutet bzw. verstanden werden sollen.

Bevor auf die Konstruktion historischer Individuen durch Wertinterpretation und Wertbeziehung näher eingegangen werden kann, muss die Kategorie des historischen Individuums in ihrer Funktionalität charakterisiert werden. Dies geschieht bereits im Roscher-Aufsatz, allerdings noch unter der Bezeichnung „Dingbegriff“. Ein Dingbegriff bringe eine „,künstliche Einheit" hervor. Es handle sich bei dieser Einheit um ein Objekt, das „empirisch ,Anschauliches“ “ enthält, aber aus der ,gegebenen Mannigfaltigkeit“ ausgewählt worden sei. Diese Auswahl entstehe „,mit Bezug auf bestimmte Forschungszwecke ,Wesentliche[s]“ ". Es handle sich also um ein „Denkprodukt [..] von nur ,funktioneller' Beziehung zum ,Gegebenen“ “. Hier liege einer der Gründe dafür vor, dass die Geschichte niemals „Reproduktion von empirischen Anschauungen“ oder „ein Abbild früherer Erlebungen“" sein könne, denn ohne Verwendung dieser Dingbegriffe im historischen Erkenntnisprozess (oder in irgendeinem anderen Erkenntnisprozess) würde es sich um ein nochmaliges Nacherleben und nicht um ein Erkennen handeln. Solange Erkenntnis das Ziel ist, arbeiten wir mit Urteilen, die ihrerseits „logische Operationen“ voraussetzen, gleichgültig um welche Wissenschaft es sich auch handeln mag. ${ }^{303}$

Erst wenn wir etabliert haben, was erkannt und erklärt werden soll, können wir mit unserer (kausalen) Deutung beginnen. Bevor wir nicht diese Voraussetzung geschaffen haben, ist uns keine Erkenntnis möglich, weil wir die ,unendliche Mannigfaltigkeit“ ja nicht einfach abbildend erfassen können, wie dies eine ,voraussetzungslose Wissenschaft“ implizieren wür-

303 Roscher-Aufsatz, 109 f. 
de. ${ }^{304}$ Es gebe eben nun einmal ,keinerlei in den Dingen selbst liegendes Merkmal, einen Teil von ihnen [sc. der Ursachen eines individuellen Gegenstandes] als allein in Betracht kommend auszusondern". ${ }^{305}$ Die Gesichtspunkte, die die Unendlichkeit des Stoffes überwinden, indem sie ihn gliedern und aus dieser Gliederung Elemente selegieren, können nicht dem Stoff selbst entnommen werden - sie sind das Ergebnis unserer Wertideen, die uns Phänomene erst zu Kulturerscheinungen werden lassen, weil Kultur, nach Weber, aus unserer anthropologischen Ausstattung stammt, zu irgendetwas Stellung zu nehmen. Stellung nehmen bedeutet, es mit Sinn/Bedeutung zu versehen, und dies wiederum kann nur geschehen, wenn wir von Werten ausgehen. ${ }^{306}$ Historische Individuen sind also die Gegenstände der Wirklichkeitswissenschaft und als solche Begriffe für individuelle, in ihrer Eigenart zu begreifende und dabei in ihrer kausalen Genese zu verstehende Gegenstände der Wissenschaft. Als Gegenstände der Wissenschaft kommen sie jedoch erst durch die begriffliche Synthese zustande, mit der die sinnlose Mannigfaltigkeit allein überwunden werden kann.

Wie geht diese Synthese genauer vor sich, das heißt, wie läuft die Wertinterpretation $\mathrm{ab}$, aus der das historische Individuum hervorgeht? Die „wertbeziehende Interpretation“ ist von zentraler Bedeutung für die Gegenstandskonstruktion und für die an sie anschließende kausale Deutung wie auch für den objektiven Charakter der Geschichtswissenschaft überhaupt. Denn sie ist es, welche die Gegenstände aus dem Bereich des Gefühlten in den Bereich des Urteils und der logischen Bearbeitung heraushebt. Sie erst legt die Grundlage für genuine Erkenntnisansprüche, die schließlich auf den Geltungsansprüchen von Urteilen basieren. Die ,wertbeziehende Interpretation“ sei nicht „Bestandteil einer [...] rein empirisch-historischen d. h. konkrete, ,historische Individuen` zu konkreten Tatsachen zurechnenden - Darstellung, sondern vielmehr [...] Formung des , historischen Indivi-

304 OA, 177.

305 OA, 177. Das Ergebnis „,voraussetzungsloser Wissenschaft“ wäre ein „Chaos von ,Existenzialurteilen“ “, ja nicht einmal dies, weil „die Wirklichkeit jeder einzelnen Wahrnehmung bei näherem Hinsehen ja stets unendlich viele Bestandteile [zeigt], die nie erschöpfend in Wahrnehmungsurteilen ausgesprochen werden können."

306 OA, 181. 
duums “ ".307

Die Wertinterpretation ist eine geschichtsphilosophische Leistung, die der historischen Erklärung vorausgeht, weil sie allererst zeigt, welche „Werte“ in einem Objekt „,verwirklicht“ sein können und welche wir dann in einzigartiger, also individueller Form in den Objekten auch tatsächlich vorfinden. Das bedeutet, dass die Wertinterpretation das, „was wir dunkel und unbestimmt, fühlen', entfalte[t] und in das Licht des artikulierten ,Wertens' erheb[t]“". Damit ist nicht gemeint, dass das Objekt bewertet wird, sondern, dass sie „Möglichkeiten von Wertbeziehungen des Objektes“ ,analysierend ,suggeriert" “.308 Es gehe darum, „uns eben die möglichen „Standpunkte' und ,Angriffspunkte' der ,Wertung' aufzudecken“. Teil dieser „Wertanalyse“ muss aber die historische Situation sein, in der das betreffende Objekt entstanden ist. Damit wird die Wertinterpretation zur „Wegweiserin“ der kausal-historischen Deutung. ${ }^{309}$ Erst indem die verschiedenen Wertbeziehungen aufgewiesen werden, kann die kausale Deutung, i. e. der Nachvollzug der historisch-genetischen Entstehung des dann herausgegriffenen Aspekts des Objekts, überhaupt erst sinnvoll beginnen: „Die Analyse jener [sc. der Wertinterpretation] wies die ,gewerteten“ Bestandteile des Objekts auf, deren kausale ,Erklärung' das Problem dieser [sc. der kausalen Deutung] ist, jene schuf die Anknüpfungspunkte, an denen der kausale Regressus sich anspinnt, und gab ihm so die entscheidenden ,Gesichtspunkte' mit auf den Weg, ohne welche er ja ohne Kompaß ins Uferlose steuern müßte. ${ }^{\text {" }} 10$ Erst die singuläre Wertinstantiierung in einem Objekt macht dieses zu einem historischen Individuum und das bedeutet, zu einem „Objekt historischer ,Erklärung “ “.311

Weber gesteht zu, dass die Wertinterpretation irreduzibel ,subjektivistisch"ist. ${ }^{312}$ Die Gegenstandskonstituierung ist von den jeweils im Histori-

\footnotetext{
307 Roscher-Aufsatz, 122.

308 Kritische Studien, $245 \mathrm{f}$.

309 Kritische Studien, $250 \mathrm{f}$.

310 Kritische Studien, 251.

311 Roscher-Aufsatz, 122.
}

312 „Sie [sc. die „wertbeziehende Interpretation“] ist in der Tat ,subjektivierend“, wenn nämlich darunter verstanden wird, daß die ,Geltung ' jener Werte selbstverständlich von uns niemals im Sinn einer Geltung als empirischer, Tatsache' ge- 
ker anzutreffenden Werten abhängig. Nur die Werte, die er kennt, ob er sie nun ablehnt oder anerkennt, kann er auch im Objekt ,entdecken'. Und aus dieser „,subjektiv“ limitierten Auswahl selegiert er erneut einen oder mehrere Wertgesichtspunkte, aus dem bzw. aus denen die historischen Individuen synthetisiert werden.

An dieser Stelle tritt uns das Objektivitätsproblem entgegen, denn die „kulturwissenschaftliche Erkenntnis in unserem Sinn ist also insofern an ,subjektive" Voraussetzungen gebunden, als sie sich nur um diejenigen Bestandteile der Wirklichkeit kümmert, welche eine - noch so indirekte - Beziehung zu Vorgängen haben, denen wir Kulturbedeutung beilegen“"313. Empirische Wissenschaften setzen logische Operationen voraus, die wiederum auf begriffliche Arbeit - zunächst und vor allem auf historische Individuen als Gegenstandssynthesen - notwendig angewiesen sind. Nun ist diese begriffliche Arbeit kein unkontrollierter Prozess, sondern eine mal mehr, mal weniger gelenkte Leistung des Erkenntnissubjekts. Der ,Stoff“, das ,Material' gibt uns nicht unsere Begriffe und unsere Erkenntnisse vor, sondern wir synthetisieren aus dem ,Gegebenen' Gegenstände heraus - und dies nicht einfach kontingenterweise oder weil wir uns dann und wann dafür, manchmal aber auch dagegen entscheiden könnten, sondern weil wir gar keine andere Möglichkeit haben, kulturwissenschaftliche Erkenntnis, also begriffsverwendende Urteile, zu erlangen. Dass diese Wertideen „subjektiv“ sind, gesteht Weber zu. ${ }^{314}$ Aber daraus folge nicht, dass die Kulturwissenschaft nur „subjektive“ Ergebnisse hervorbringe, denn in der Methode, i. e. der Anwendung der ,begrifflichen Hilfsmittel“, sei - im Gegensatz zur Abhängigkeit der Gegenstandkonstituierung und der Selektion von Ursachen von „Wertideen“, wie auch der Bildung von „begrifflichen Hilfsmitteln“ (i. e. Idealtypen) von den „Gesichtspunkten“ - der Forscher an die „Normen unseres Denkens gebunden“, die für alle gelten, die „Wahrheit wollen". 315

Ob diese Normen nun tatsächlich für alle gelten, ist, abgesehen von eini-

meint sein kann." (Roscher-Aufsatz, 122)

313 OA, 182.

314 OA, 183.

315 OA, 184. 
gen basalen logischen Gesetzen, durchaus zu bezweifeln. Webers Verweis auf sie sollte besser durch die oben skizzierten Restriktionen der konstruktiven Willkür des Historikers ersetzt werden. Grundsätzlich jedoch muss sich jeder konstruktionistische Versuch einer Vereinbarung von Standortgebundenheit („subjektiven Einflüssen“ mit Weber) mit Objektivität der Herausforderung stellen, der individuellen (und auch kollektiven) Konstruktionswillkür Restriktionen aufzuerlegen. Andernfalls wäre der Einwand, ob es sich bei solcherart begrifflichen Konstruktionen nicht um eine illegitime Aufoktroyierung anachronistischer Begriffe handelte, ${ }^{316}$ tatsächlich schlagend. Eine ausgearbeitete konstruktionistische Theorie müsste hier eingehend und sorgfältig argumentieren (eingehender und sorgfältiger als es hier die knappen Grundzüge erlauben).

Obschon damit die Grundlage kulturwissenschaftlicher Gegenstandkonstituierung „subjektivistisch“ ist, hebt die wertbeziehende Interpretation historische Erkenntnis erst in den Rang potentiell objektiver Erkenntnis, weil die wertbeziehende Interpretation propositional ist, während das wertende Nacherleben oder Nachvollziehen eine rein emotionale Angelegenheit bleibt. Da auch für Weber Objektivität der Erkenntnis zukommt, ist die Satzförmigkeit notwendige Bedingung von Objektivität, ${ }^{317}$ und damit ist die subjektivistische Wertinterpretation notwendige Bedingung von Objektivität. Grundlage der Wertinterpretation bleiben aber die jeweiligen Werte des Kulturwissenschaftlers, die er im ersten Schritt überhaupt erst potentiell dem Gegenstand zuschreiben kann und die er dann, im zweiten Schritt, im Gegenstand aktualisiert findet. Erst wenn er eine individuelle Konstellation der Wertverwirklichung in einem Objekt vorgefunden hat, ist er im Besitz eines „historischen Individuums“, das Objekt seiner historischen Erklärung ist. Aber: Die Zurechnung von Ursachen zu historischen Individuen „wird mit dem prinzipiellen Ziel vorgenommen, ,objektiv‘ als Erfahrungswahrheit gültig zu sein mit derselben Unbedingtheit wie irgendwelche Erfahrungserkenntnis überhaupt, und nur die Zulänglichkeit des Materials entscheidet über die, nicht logische, sondern nur faktische Frage, ob

316 Vgl. Abs. 3.1.

317 Roscher-Aufsatz, 123. Vgl. die etwas ausführlichere Parallelstelle in Kritische Studien, $252 \mathrm{f}$. 
sie dies Ziel erreicht, ganz ebenso wie dies auf dem Gebiet der Erklärung des konkreten Naturvorgangs der Fall ist. ,Subjektiv' in einem bestimmten Sinn ist nicht die Feststellung der historischen ,Ursachen' bei gegebenem Erklärungs-,Objekt', sondern die Abgrenzung des historischen ,Objektes ${ }^{6}$, des ,Individuums' selbst, denn hier entscheiden Wertbeziehungen, deren ,Auffassung' dem historischen Wandel unterworfen ist. ${ }^{\text {“318 }}$

Weber legt damit eine Theorie historischer Gegenstandskonstruktion vor, welche die Unverzichtbarkeit des Standortes in der Überbrückung des hiatus irrationalis fundiert. Hier muss das Besondere an dieser Problemanalyse für die vorliegende Arbeit hervorgehoben werden. Weber stellt nicht einfach auf die Unendlichkeit der Ursachen eines Ereignisses ab, die uns dazu zwingt, Ursachen oder Bedingungen zu selegieren. (Eine Argumentation, die häufig anzutreffen ist, wenn es darum geht, die Perspektivität historischer Erkenntnis zu belegen.) Dass jeder Historiker dies tun muss und dass dieser Selektionszwang aufgrund unendlicher Kausalreihen von vornherein jeden Abspiegelungsanspruch zunichte macht, wird zwar auch von Weber in den Passagen zur Gegenstandskonstruktion angegeben. Darin allerdings erschöpft sich Webers Argumentation nicht. Weber setzt die Notwendigkeit historischer Perspektivität tiefer an. Um Ursachenselektion überhaupt erst betreiben zu können, müssen wir aus den begrifflich noch gar nicht adäquat bearbeiteten Daten erst Gegenstände herauspräparieren. Erst dann sind wir dazu in der Lage, überhaupt sinnvoll Ereignisse aus der Unendlichkeit potentieller und faktisch kausal relevanter Bedingungen auszuwählen. Ohne die auf ,subjektiven“ Voraussetzungen basierende Gegenstandsbildung gibt es gar kein Kriterium dafür, was Ursache sein kann. Anders formuliert: Ohne das Explanandum konstruiert zu haben, kann kein Explanans aufgesucht werden.

Aber auch diese Ebene der Explanandumskonstruktion stellt noch nicht die basalste Schicht dar, auf welcher der Standort des Historikers einschlägig an der Gegenstandskonstruktion beteiligt ist. Die kognitive Verarbei-

318 Kritische Studien, 261. Vgl. OA, 184: „Endlos wälzt sich der Strom des unermeßlichen Geschehens der Ewigkeit entgegen. Immer neu und anders bilden sich die Kulturprobleme, welche die Menschen bewegen, flüssig bleibt, [sic] damit der Umkreis dessen, was aus jenem stets gleich unendlichen Strome des Individuellen Sinn und Bedeutung für uns erhält, ,historisches Individuum‘ wird.“ 
tung unstrukturierter Datenmengen kann nur begrifflich vor sich gehen das gilt, laut Weber, sowohl für die Kultur- als auch für die Naturwissenschaften. Beide müssen Begriffe entwickeln, mit deren Hilfe Daten strukturiert werden können. Die Naturwissenschaften sind dazu in der Lage, generelle Begriffe zu entwickeln, unter die weitere Individuen subsumiert werde können, ${ }^{319}$ die Kulturwissenschaften dagegen müssen auf ihre wertinduzierten Gegenstandsbegriffe zurückgreifen. Sie haben keine andere Möglichkeit, Gegenstandsbegriffe zu bilden. Ohne Gegenstandsbegriffe haben die Kulturwissenschaften aber keine Möglichkeit, Erkenntnis zu erlangen, denn ohne sie fehlt ihnen die Fähigkeit, propositional zu werden. Ohne standortabhängige Gegenstandskonstruktion gibt es keine Möglichkeit, Propositionen zu bilden und damit Erkenntnis zu erlangen.

$\mathrm{Ob}$ die kulturwissenschaftlich-historische Gegenstandskonstruktion nun genau so vor sich gehen muss, wie Weber dies konzipiert hat, kann durchaus in Einzelheiten bezweifelt werden. ${ }^{320}$ Immerhin liegt aber in Webers

$319 \mathrm{Ob}$ dies für die Naturwissenschaften genau so zutrifft, mag dahingestellt bleiben.

320 Zum einen kann bezweifelt werden, ob ,singuläre Begriffe“ nicht eine contradictio in adiecto sind und damit Webers WL in ihrem semantischen Kern bereits flugunfähig ist, bevor sie überhaupt die Flügel ausbreiten konnte. Zum anderen kann und muss weiter gefragt werden, woher die Werte kommen, die nun an dieser fundamentalen Stelle in Webers Theorie so prominent fungieren. Einen Wertobjektivismus vertritt Weber dezidiert nicht. Für ihn können Werte nur existentiell-dezisionistisch angenommen oder abgelehnt werden. Einem (wissenschaftlichen) Beweis für die Geltung eines Wertes gegenüber einem anderen steht Weber mehr als nur skeptisch gegenüber - jeder müsse eben selbst den Dämon finden, der seines Lebens Fäden in Händen hält. Damit würde, denkt man diesen Ansatz weiter, jede historische Narration ihren Ausgang (ihr Explanandum) und sicherlich auch einiges an explanatorischem Material (die Explanantia) von einem restlos subjektiven Fundament aus erfolgen, was es mit sich bringen würde, dass sich die fachdisziplinäre Auseinandersetzung nicht mehr um objektiv und nicht-objektiv oder wahr und falsch oder Beweis und Nicht-Beweis drehen würde, sondern lediglich um die evaluative Zurückweisung von Gegenstandskonstruktionen womit eine sehr enge Analogie zu Ankersmits Schlussfolgerung bestehen würde, dass der Streit über den Objektivitätsgehalt historischer Narrationen sinnlos sei, weil ja ohnehin alle Narrationen analytisch wahr seien, jeder also seine eigene Definition einer narrative Substanz vorlegt, sobald er eine historische Narration vorlegt. Man kann mit einigem Recht skeptisch sein, ob Webers Theorie einer 
WL ein recht detailliert ausgearbeiteter Vorschlag verborgen, an den sich anknüpfen und der sich ausarbeiten und verbessern ließe. An ihm sollte sich jeder konstruktionistische Versuch abarbeiten, Standortgebundenheit mit Objektivität zu vereinbaren. Hier soll aber nur auf einige Punkte aufmerksam gemacht werden, die in Webers „Wissenschaftslehre“ keine Aufmerksamkeit erhalten haben.

Sind solche Gegenstandkonstruktionen vielfach Konstruktionen des Historikers, so sind sie mindestens ebenso häufig auch Importe aus Nachbardisziplinen oder aus Begriffsrahmen und Gegenstandssytemen des Alltags. Das Mittelalter als Periode ist eine historische Konstruktion, der „Westfälische Frieden“ oder der „Vertrag von Versailles“ dagegen sind Importe aus den politischen, diplomatischen und vielleicht auch publizistischen Bereichen allgemeinmenschlicher oder kulturspezifischer Gegenstandskonstruktion. Das bedeutet allerdings nicht, dass diese Importe keiner begrifflichen Modifikation unterworfen werden können. Ein Historiker übernimmt vielleicht die Bezeichnung „Friede“ aus den diplomatischen Noten der damals an der Aushandlung des „Westfälischen Friedens“ beteiligten Staatsmänner und Unterhändler, die Qualifikation der damals ausgetauschten und festgehaltenen Sprechakte als eines tatsächlichen Friedens kann der Historiker qua Historiker, i. e. Kenner der vergangenen Zukunft der damals Beteiligten, jedoch nach Maßgabe seiner Auffassung dessen, was „Frieden“ bedeutet, korrigieren oder bestätigen. Denn es wäre immerhin denkbar, dass damals zwar jede der beteiligten Kriegsparteien guten Gewissens einen Frieden schließen wollte, die Kampfhandlungen jedoch kein wirkliches Ende gefunden hätten. Nach Maßgabe dessen, was die begriffliche Implikation des Begriffs „Frieden“ im einschlägigen Begriffsrahmen des

derartigen Konsequenz wirklich entkommen kann, ohne objektive Werte anzunehmen (wie etwa Rickert [vgl. dazu die ersten beiden Kapitel aus Burger 1987]) oder sich, wie das hier geschehen soll auf die begrifflichen und doxastischen Restriktionen zu berufen, welche die Begriffsrahmen respektive das Überzeugungssystem mit sich bringen. Eine durchgeführte Dienstbarmachung von Webers WL müsste detaillierter herausarbeiten, wo sowohl der (wertinduzierten) Gegenstandskonstruktion als auch der verstehenden Deutung Grenzen gesetzt sind, die der Historiker nicht mehr überschreiten kann, ohne nur noch zu sich selbst zu sprechen. 
Historikers ist, handelte es sich dann um keinen wirklichen Frieden. Der Gegenstandsimport „(Westfälischer) Friede“ würde, nach dem Begriffsrahmen des Historikers also auf keinen wirklichen Gegenstand, auf kein genuines Segment der Geschichte referieren. Erst die historische Retrospektion zusammen mit den gegenwärtigen Begriffsrahmen des Historikers erlauben es, die Geschichte mit historischen Individuen zu bevölkern.

Teil der Perspektive sind demnach immer auch begriffliche Neuerungen (und damit: Gegenstandskonstruktionen) der Nachbardisziplinen und der Alltagssprache. Mit dem Wandel der dort beheimateten Begriffsrahmen können sich auch die Begriffsrahmen und damit die Ontologie der Geschichte wandeln. ${ }^{321}$ Und dass sich andere Wissenschaften nicht nur in Form eines Erkenntniszugewinns, sondern auch methodisch-begrifflich wandeln, wird wohl kaum in Abrede gestellt werden können. Ebenso wenig wird in Abrede gestellt werden können, dass sich die Begriffsrahmen der Geschichte wandeln.

\subsubsection{Die Narrationsbildung}

Ist aber erst einmal ein Gegenstand der Betrachtung konstruiert oder importiert, steht der Historiker vor der Aufgabe, eine Narration zu konstruieren, um den historischen Wandel des konstruierten historischen Individuums über einen bestimmten Zeitraum hinweg zu verfolgen. Dass mit der standortabhängigen Konstruktion des Protagonisten einer historischen Narration bereits die Perspektive des Historikers notwendig ins Spiel kommt, ist gezeigt worden, aber auch auf der Narrationsebene selbst wird deutlich, dass historische Erkenntnis nicht ohne Perspektive möglich ist. Wie dieser „subjektive“ Einfluss in diesem Zusammenhang aussieht, wird hier kurz

321 Jedem, der näher mit der Entwicklung der Gesellschafts- oder Sozialgeschichte vertraut ist, wird die ungeheure Bedeutung soziologischer Begriffs- und Theoriebildung für diese Gattung der Geschichtswissenschaft vertraut sein. 
skizziert. Dazu werden einige Elemente des minimalistischen Narrativismus verwendet werden müssen.

Patzig hat gegen eine korrespondenztheoretisch aufgefasste Objektivität zu bedenken gegeben, dass Abbildung gut und schön sei, Wissenschaft aber eben nicht an allen möglichen trivialen Wahrheiten interessiert sei, sondern nur an einigen ausgesuchten. ${ }^{322}$ Es müsse also ein Relevanzkriterium für die jeweiligen Wahrheiten geben, die dargestellt werden. Genau diese Leistung kann ausschließlich von einer perspektivischen Geschichtstheorie erbracht werden, und hier ist es die minimalistische Narrationstheorie, die über die narrative Kohärenz ein Relevanzkriterium für historische Erkenntnis beisteuert. ${ }^{323}$

Narrative Kohärenz, so wurde im dritten Kapitel gesagt, entsteht dadurch, dass die Eigenschaftsveränderung eines historischen Individuums als Protagonisten der historischen Narration als Kriterium dafür hergenommen wird, welche der Aussagen, die potentiell für eine narrative Integration zur Verfügung stehen, in eine Narration aufgenommen werden können. Ihre Funktion als Elemente der Beschreibung einer Eigenschaftsveränderung und der interaktionelle Zusammenhang, in dem sie untereinander stehen, , verklammert‘ diese Aussagen zu einer Narration. Die Abhängigkeit des historischen Individuums vom konstruktiven Beitrag des Historikers ist gezeigt worden. Inwiefern aber - ist der Protagonist erst einmal konstruiert - geht die Perspektive des Historikers auch in die Narrationsbildung mit ein?

Genuine Geschichtsschreibung liegt erst dann vor, wenn der Historiker auf Ereignisse zurückblicken kann. So trivial dies scheinen mag, so folgenreich ist diese Binsenwahrheit. Sein Blick auf die Ereignisse, die er darstellen möchte, wird nicht nur von den Ereignissen bestimmt, die diesem Ereignis vorangegangen sind, sie werden ebenso von den Ereignissen bestimmt, die ihm nachgefolgt sind. Historische Darstellungen sind erst dann genuin historisch, wenn ein Ereignis mit Bezug auf die ihm nachfolgenden Ereignisse beschrieben wird. Wer also die Geschichte des Zweiten Weltkrieges erzählt, beschreibt die Ereignisse, die sich während des Zweiten

322 Patzig 1977, 325.

323 Daneben gibt es noch weitere, etwa evaluative oder methodologische. 
Weltkrieges zugetragen haben unter dem Blickwinkel zum Beispiel des Kriegsendes, aber auch unter dem Blickwinkel der darauf folgenden Ereignisse. Damit ist nicht gemeint, dass jedes Kriegsereignis so beschrieben wird, dass es mit dem „Kalten Krieg“ oder gar mit dem Zusammenbruch des UdSSR in Beziehung gesetzt wird, es ist damit gemeint, dass vergangene Ereignisse, die von der einen Darstellung als Teil einer Erzählung beschrieben wird, die vor ca. 60 Jahren an ihr Ende gekommen ist, auch so beschrieben werden können, als seien sie Teil einer Kausalkette, i. e. einer Gegenstandsveränderung, die ihr Ende erst zum Beispiel 1989 gefunden hat. (Man denke an eine Geschichte, die den Kampf „des Westens“ gegen den sowjetischen Kommunismus als Protagonisten konstruiert hat.)

Seine Position in der Geschichte bestimmt, wie der Historiker die Ereignisse, die er narrativ erfassen möchte, konstruiert, und es bestimmt sich die historische Bedeutung eines Ereignisses immer danach, was nach diesem Ereignis geschehen ist, vor allem aber, wo der Historiker das Ende der Geschichte ansetzt. In die Konstruktion des Protagonisten geht, mit andere Worten, seine ,Lebensdauer' ein. Teil seiner Identifikationsbedingungen, die es erlauben, ihn als historisches Ereignis (also als eine in der Zeit erstreckte Entität) zu identifizieren, ist also seine zeitliche Erstreckung, die durchaus je nach den Voraussetzungen des Historikers verschieden sein können. (Eine Schlacht etwa kann konzipiert werden als Ereignis vom ersten bis zum letzten Schuss oder vom Marschbefehl bis zum Rückzugsbefehl, von der Truppenaufstellung bis zur wirren Flucht einer der Parteien etc.) Die Anfangs- und Endpunkte der Narration geben die ,Lebensdauer an, wobei die zur Narration gehörenden Ereignisse in Hinsicht auf dieses Ende und die zwischen Anfang und Ende liegenden Ereignisse narrativ integriert werden. Die Wahl dieser Punkte bestimmt damit über die Selektion der zugehörigen Aussagen. Sie liegt aber beim Historiker.

Doch nicht nur die bloße Selektion von Aussagen (oder Ereignissen) bestimmt sich durch die Setzung des Anfangs- und Endpunktes und die Konstruktion des historischen Individuums. Auch die narrative Rolle eines Ereignisses kann erst dann bestimmt werden, wenn das historische Individuum konstruiert worden ist, was im Fall eines historischen Protagonisten auch seine ,Lebensdauer' einschließt. Wie Danto gezeigt hat, kann die kau- 
sale oder funktionale Rolle eines Ereignisses nur relativ auf die folgenden Ereignisse festgestellt werden. Dabei mag es sich insofern um eine Binsenwahrheit handeln, als ein Ereignis in der Regel nur Ursache von etwas sein kann, das nach ihm stattfindet, weil aber die Kausalkette, welche die Ereignisse untereinander verbindet, niemals abreißt, kann von einem Ereignis nur relativ zu einem anderen als Endpunkt bestimmten Ereignis ein kausaler Aspekt als Erklärung herausgegriffen werden. ${ }^{324}$ Das Ursachen-Ereignis trägt seine kausalen Eigenschaften nicht auf der Stirn geschrieben.

Je nach der kausalen (oder funktionalen etc.) Rolle, die einem Ereignis in Relation zum Protagonisten zugedacht ist, wird dieses Ereignis als Sachverhalt in Form einer Proposition konstruiert werden. Die ,Tatsachen' (i. e. die Propositionen, die Sachverhalte ausdrücken und im Modus assertorischer Rede behauptet werden) werden damit narrations- und damit perspektivenrelativ aus den Quellen herausgelesen. Darin liegt der Grund dafür, dass die Vorstellung, eine Quelle berge bestimmte ,Tatsachen' in sich, die der Historiker herausarbeite, nicht ganz den Kern der Sache trifft. Die Quelle birgt eine unendliche Anzahl von potentiellen ,Tatsachen' in sich, die je nach Konstruktion des historischen Individuums als Protagonisten und der Narration aus ihr herausgelesen werden. Andere, Tatsachen' bleiben im Dunkeln und können erst nach einem Perspektivenwandel entdeckt werden.

Dabei sind Selektion und Beschreibung der Ereignisse nicht einfach dem willkürlichen Postulieren von Endpunkten oder der Konstruktion von historischen Individuen ausgeliefert. Der interaktionelle Zusammenhang, in dem die Aussagen stehen, liefert eine Mikrostruktur historischer Narrationen, die beschränkt, welche Aussagen Teil der Narration werden und wie

324 Dass selbst dann noch eine unendliche Anzahl von Ursachen (oder genauer: notwendigen Bedingungen) eines Ereignisses zu erzählen wären, also auch eine noch stärkere Einschränkung dessen vorgenommen werden muss, was als relevant zu gelten hat, ist selbstverständlich. Auch an dieser Stelle werden es mal wisssenschaftlich-theoretische Überzeugungen sein, die Relevanzkriterien setzen, mal werden es triviale Alltagsweisheiten sein. (Dabei ist ein nicht unerheblicher Erkenntnisgewinn in der Geschichte dadurch erzielt worden, dass die Binsenweisheiten des Alltags kritisch hinterfragt und durch wissenschaftliche Theoriebildung ersetzt wurden.) 
sie $\mathrm{zu}$ formulieren sind. Bestimmte Festlegungen (metaphysischer, theoretischer oder auch begrifflicher Art) des Historikers, die er im Einklang mit seinen Begriffsrahmen und dem übrigen Überzeugungssystem getroffen hat, lassen bestimmte Möglichkeiten , sichtbar ${ }^{6}$ werden, andere dagegen gar nicht erst auftauchen oder, falls sie ihm dennoch begegnen, als unwahrscheinlich erscheinen. Hier ist er in seiner Willkür sogar weiter eingeschränkt, weil die Quellen ein Vetorecht besitzen (das fachdisziplinär überwacht und durchgesetzt wird). Wie er seine ,Tatsachen' konstruiert, das heißt präziser, welche Tatsachen er in seinen Propositionen wie ausdrückt, und welche er dann für seine Narration auswählt, hängt von der Konstruktion des historischen Individuums als Protagonisten und hier speziell der ,Lebensdauer', die er als eine Identifikationsbedingung in die Konstruktion einbringen muss, zusammen. Diese Konstruktionen sind von seiner eigenen Position in der Geschichte und seinen theoretischen, metaphysischen, evaluativen Voraussetzungen, aber auch von seinem Erkenntnisinteresse abhängig.

\subsubsection{Theoretische Vorannahmen, Metaerzählungen und Erkenntnisinteresse}

Was aber liefert Kriterien für die Konstruktion eines historischen Individuums auf die eine und nicht auf eine andere Art? Was schränkt die immer noch gewaltige Anzahl an narrativ integrierbaren Ereignissen (,Tatsachen") noch weiter ein, als es die narrative Mikrostruktur und das historische Individuum bereits tun? Eine Antwort ist die nächstliegende und klassische: das Erkenntnisinteresse des Historikers. Das Erkenntnisinteresse bestimmt mit, welche Ereignisse seine Aufmerksamkeit verdienen, sei es als Explananda, sei es als Teile der Erklärung. Doch woraus speist sich sein Erkenntnisinteresse?

Neben bestimmten wissenschaftstaktischen Erwägungen - welches Thema ist noch nicht oft bearbeitet worden; wo lassen sich innovative Ergeb- 
nisse erzielen; welches Thema wird in Zukunft in Mode kommen? - dürften dies Wertüberzeugungen sein. ${ }^{325}$ Ein Ereignis, ein potentielles historisches Individuum muss von einem gewissen Erkenntniswert für den Historiker und die Gesellschaft, in der er lebt, sein. Der Erkenntniswert bestimmt sich seinerseits darüber, welche Werte in der Gesellschaft gelten und welche er selbst teilt. Soll zum Beispiel eine demokratische Gesellschaftsordnung verteidigt werden, weil demokratische Werte in der Gesellschaft gelten und vom Historiker geteilt werden, dann kann es wert sein, die totalitären Ursprünge dieser Gesellschaft mit all ihren damaligen Verbrechen darzustellen.

Gesellschaftliche Werte werden u. a. immer noch in sog. ,großen Erzählungen ${ }^{\text {“326 }}$ und spekulativen Geschichtsphilosophien festgehalten und weitergegeben. Sie bilden immer noch den Verständigungshorizont, der zumindest für weite Teile einer Gesellschaft akzeptabel und die Voraussetzung für basalen gesellschaftlichen Konsens ist. Die „Entzauberung“ der Welt, das Fortschreiten oder die „Dialektik“ der Aufklärung, der Kampf zwischen Freiheit und Totalitarismus, Geschichte als Klassenkampf und ande-

325 Hier liegt der Anschluss an Webers „Wissenschaftslehre“ auf der Hand, denn letztlich bestimmt das wertinduzierte Erkenntnisinteresse die Konstruktion der historischen Individuen und der Idealtypen.

326 Wenn Lyotard auch das „Ende der großen Erzählungen“ prognostiziert, wo nicht konstatiert haben mag, ganz kann wohl kaum auf irgendwelche (peudo-)historischen Rahmenerzählungen verzichtet werden, in denen die grundlegendsten Werte und Selbstverständigungen einer Gruppe oder einer Gesellschaft verankert werden. Selbst das postmoderne „Ende der großen Erzählungen“ ist nur eine weitere „große Erzählung“, wenn sie sich auch den Anschein einer besonders offenen Metaerzählung gibt. Dabei haben Globalisierung, die Dezentrierung vormals strikt euro-zentrischer Perspektiven, aber auch die politischen Katastrophen des 20. Jahrhunderts die Uniformität von Metaerzählungen und von metaphysischen Präsuppositionen gebrochen. Diese Erkenntnis hat sich aber nicht erst mit dem von ,der Postmoderne“ verkündeten „Ende der großen Erzählungen“ eingestellt. Z. B. hat schon einige Jahre früher, im Jahr 1968, Georg Iggers das Ende des deutschen Historismus und seiner metaphysisch-philosophischen Grundlagen nach dem Ersten Weltkrieg, vor allem aber nach dem Ende des „Dritten Reiches“ nachgezeichnet (Iggers 1997, Kap. VIII Der Verfall der deutschen, Geschichtsidee'. Der Einfluß der beiden Weltkriege und des Totalitarismus auf das deutsche Geschichtsdenken). 
re große Erzählungen sind wertbestimmende Faktoren des historischen Erkenntnisinteresses und damit erkenntniskonstitutive Elemente des jeweiligen Standortes. An dieser Stelle sind es natürlich gerade die Ergebnisse der Geschichtsschreibung, die ihrerseits auf die Werte zurückwirken. Der Verlauf der Geschichte oder genauer: das Bild von der Vergangenheit, das von den Historikern vermittelt wird, bestimmt wiederum, welche großen Erzählungen möglich sind und welche bereits an den historischen Fakten scheitern.

Von entscheidender Bedeutung für das Erkenntnisinteresse des Historikers sind neben seinen Wertvorstellungen auch seine theoretischen Vorannahmen. Auch sie bestimmen, was einen Anteil an seinem Aufmerksamkeitshaushalt erhalten kann und soll. Sie bestimmen sein Erkenntnisinteresse dadurch mit, dass sie bestimmte Fragestellungen als fruchtbar, andere dagegen als wenig aussichtsreich erscheinen lassen. Sie bestimmen aber auch, welche Entitäten der Historiker zu ,sehen' in der Lage ist, mithin geben sie der Datenmenge Kontur, und natürlich bestimmen sie seine Methoden mit. So könnte es zwar durchaus von Interesse sein, die „Industrielle Revolution“" als eine wirtschaftliche Entwicklung zu betrachten, die allein von den genialen Erfindungen einiger herausragender Ingenieure und Experimentatoren ausgelöst worden ist. Sein (wirtschafts-)theoretisches Wissen wird ihm aber sagen, dass eine solch durchgreifende sozio-ökonomische Umwälzung nur dann möglich sein kann, wenn kulturelle, wirtschaftliche und auch soziale Strukturen und Veränderungen ineinander greifen. Ohne begünstigende Rahmenbedingungen und Veränderungen im Rechtsund Bildungswesen und der Wirtschaftsordnung kann keine solche „Revolution" vonstatten gehen, und entsprechend wird er seine Methoden, die im ersten Fall vielleicht nur daran orientiert sind, die Motivlage einiger weniger Persönlichkeiten darzustellen, dahingehend abwandeln müssen, dass nun auch sozio-ökonomische und kulturelle Strukturen Teil seiner Narration werden können, wie er auch eine Ontologie gelten lassen muss, die zum Beispiel gesellschaftliche Strukturen oder kausal wirksame kulturelle Faktoren jenseits des individuellen Wollens einiger Akteure kennt. Seine theoretischen Vorannahmen bringen damit Methoden in seinen Standort ein, aber auch Ontologien und aussichtsreiche Forschungsfragen. 


\subsubsection{Die Metareflexion}

Wie aber kann Ordnung in dieses zumindest prima facie chaotische Hin und Her von Begriffsrahmen, theoretischen Vorannahmen, Importen aus Nachbardisziplinen, Erkenntnisinteressen, Methoden, Ontologien etc. gebracht werden? Oder muss man annehmen, dass alle diese Elemente ihre Funktionen im Erkenntnisprozess ohne Kontrolle durch den Historiker versehen?

Ordnung und Einflussnahme bringt der Historiker in diese verschiedenen Elemente, indem er sich reflexiv zu ihnen verhält. Er bringt sich in eine reflektierende Metaposition, von der aus er die Elemente des Standortes wissenschaftstheoretisch-epistemologisch beurteilt und je nach Ergebnis dieser Metareflexion Element für Element ersetzt oder modifiziert.

Was sind die Kriterien, nach denen er seine Beurteilung abgibt? Einfach gesagt, handelt es sich um seine Überzeugungen davon, was (gute) Wissenschaft ausmacht. Allein in diesem, vielleicht nicht sehr anspruchsvollen Sinn kann gesagt werden, dass es sich dabei um einen wissenschafts- und erkenntnistheoretischen Prozess handelt. Ausschlaggebend dürften in erster Linie nicht vom jeweiligen Historiker unmittelbar rezipierte philosophische Wissenschaftstheorie und Epistemologie sein, sondern vielmehr während seiner wissenschaftlichen Ausbildung antrainierte Beurteilungsstandards. Ob diese Standards später hinterfragt und aus wissenschaftlich-rationalen Beweggründen anerkannt bzw. abgelehnt werden oder ob es sich um unhinterfragte ,Abrichtung' oder ob es sich dabei um erfolgversprechende, ,gute' Standards und Kriterien handelt, mag dahingestellt bleiben - an ihrer Funktionsweise ändert sich nichts. Natürlich werden im günstigsten Fall wissenschaftstheoretische, epistemologische, aber auch methodologische Erkenntnisse historischer Nachbardisziplinen - darunter auch gerade der Philosophie - eine große Rolle spielen, sei es auch nur mittelbar in Form sekundärer Rezeption einer Auseinandersetzung unter seinen Kollegen oder eines Theoriereferats von dritter Seite, ausgeschlossen ist aber die nicht weiter hinterfragte Beibehaltung der einmal ansozialisierten Standards in Form von selbstverständlichen, erprobten ,Wahrheiten' keines- 
wegs.

So beschrieben ist die Fähigkeit zur Reflexion auf die Elemente des Standortes kein Bestandteil der unmittelbar erkenntnismitbestimmenden Perspektive mehr, sondern eine Fähigkeit, die zwar enormen Einfluss auf die Zusammensetzung des Standortes (und damit auf die historische Erkenntnis) besitzt, dabei aber gewissermaßen über ihr schwebt und daher nicht mehr unmittelbar auf die Erkenntniskonstruktion einwirkt. Sie wirkt aber mittelbar darauf ein, weil durch sie die erkenntniskonstruktive Perspektive analysiert und gegebenenfalls modifiziert wird.

Hier kann zu bedenken gegeben werden, dass selbst eine Metareflexion auf die eigenen Voraussetzungen keine wirklich rationale Ausweisung der eigenen Perspektive leisten könne. Allenfalls könnten gewisse Idiosynkrasien, Parteilichkeiten oder auch Gruppenrelativismen abgemildert werden, weil gerade die am ehesten unbemerkt bleibenden Voraussetzungen diejenigen seien, die den größten Einfluss auf die Arbeit des Historikers haben. ${ }^{327}$

Gegen dieses Bedenken können zwei Erwiderungen vorgebracht werden. Erstens beachtet dieser Einwand die soziale Dimension des wissenschaftlichen Diskurses zu wenig. Die Rechenschaft, die über die jeweiligen individuellen Voraussetzungen abgelegt werden muss, kann der einzelne Wissenschaftler nicht allein in foro interno in aller Abgeschiedenheit leisten. Er wird von seinen Kollegen und anderen Rezipienten seiner Arbeit darauf hingewiesen werden, an welchen Stellen seiner Arbeit sich - trotz aller vorgängiger individueller Bemühungen seinerseits - der „Parteimann“ zu Wort gemeldet hat. Für das jeweils in Frage kommende Werk mag dieser Hinweis zu spät kommen, für seine weiteren Forschungen dagegen nicht (sofern er sich überhaupt auf das Spiel der Metareflexion einlässt - was ihn, sollte er es nicht tun, desto offensichtlicher als parteilich erweist).

An dieser Stelle wird der Rationalitätsdruck durch eine historische Entwicklung noch erhöht, die den Trend zur Metareflexion noch verstärkt. Es kann nämlich immer noch die Gefahr einer ethnozentrisch-kulturrelativen Parteilichkeit bestehen. Dann würde auch die Kontrolle, die durch die Fachkollegen und andere Zeitgenossen ausgeübt wird, nicht genügen und

327 So Mommsen 1977, 452. 
zwar nicht aus böser Absicht, sondern weil - ganz im Sinne des Einwandes - die eigenen kulturellen Voraussetzungen so unbemerkbar tief verankert sind, dass sie von allen Diskursteilnehmern übersehen werden. Genau dieser Effekt hat lange Zeit vorgeherrscht, wird aber nun mehr und mehr durch die Verbreitung der wissenschaftlichen Beschäftigung mit der Vergangenheit in der ganzen Welt zurechtgerückt. Die Anstrengungen post-kolonialer Geschichtsschreibung in Afrika oder Asien sind dafür gute Beispiele. $^{328}$

Zweitens unterschätzt dieser Einwand die Leistungen wissenschaftlicher Anstrengungen anderer Fachbereiche, die es - sofern sie vom Historiker aufgenommen werden - ermöglichen, sich über die eigenen Voraussetzungen aufzuklären. Dazu zählen die Ergebnisse von Disziplinen wie der Ethik, der (internationalen) Politikwissenschaft oder der Soziologie, die den Blick auf fremde wie eigene Blickverengungen freigeben. ${ }^{329}$

In der Metareflexion ist denn auch die Möglichkeit zu einer konstruktionistischen historischen Objektivität am nachdrücklichsen verankert, weil durch sie der Standort des Historikers zu mehr als bloß einer subjektiv vorausgesetzten, , unhintergehbaren' Erkenntnisbedingung wird. Denn an dieser Stelle tritt am deutlichsten die aktivische Komponente des Konstruktionismus in den Blick. Auf dieser Ebene ist es dem Historiker möglich, alle die Parameter seines Erkenntnisprozesses zu überdenken, mit dem fachdisziplinären Konsens abzugleichen (und dann womöglich von diesem abzugehen) und seinen Standort aktiv gemäß seiner Einschätzung von wissenschaftlicher Rationalität und Objektivität auszurichten. Es handelt sich um die Fähigkeit, nach Maßgabe der Vernunft und seiner Auffassung von wissenschaftlicher Redlichkeit, die Mittel zu wählen, um dem Ziel am nächsten zu kommen, die Vergangenheit so darzustellen, „wie es eigentlich gewesen“. Dass es sich bei dieser Fähigkeit um eine geschichtsunspezifische

328 Zur Entwicklung der Geschichtswissenschaft in der sog. „Dritten Welt“ und den ehemaligen Kolonialgebieten der europäischen Kolonialmächte vgl. Raphael 2003, 51-57 und 249-256.

329 Dass es sich hierbei um ein idealisiertes Bild einer in the long run zunehmenden Verständigung und Rationalität handelt, die sogar einer regressiven Rationalitätsdynamik zu Opfer fallen kann, ist bereits thematisiert worden. (Vgl. 4.2.3, Anm. 217) 
Fähigkeit handelt, die man am besten als Vernunftkomponente historischer Erkenntnis auf den Begriff bringt, tut bei all dem nichts zur Sache, denn bei ihr handelt es sich um eine conditio sine qua non aller Wissenschaft. 


\subsection{Resümee und Ausblick}

Um eine vollständige Theorie perspektivischer und dabei objektiver historischer Erkenntnis vorzulegen, müssten die einzelnen hier aufgezählten Elemente des Standortes wesentlich ausführlicher analysiert werden. Daneben müssten die bislang nicht oder nur en passant angesprochenen Elemente (Quellenselektion, fachdisziplinäre Institutionalisierung $u$. a.) in den Katalog mit aufgenommen werden. Außerdem konnte nur in Ansätzen die Wechselwirkung zwischen den Elementen angedeutet werden, eine abgerundete Theorie müsste aber an dieser Stelle besonders ausführlich sein. Gerade die Sonderstellung der Ebene der Metareflexion könnte in einer ausgearbeiteten Theorie besonderer Aufmerksamkeit wert sein. Hier müsste insbesondere die Trennung zwischen ihr und den ihr zur Reflexion unterworfenen Elementen noch schärfer gezogen werden.

Weiter bedürften die Restriktionen, die der konstruktiven Willkür des Historikers auferlegt werden, einer detaillierteren Analyse. Allein die „Normen des Denkens", das wissenschaftliche Globalziel, die Wahrheit ans Licht bringen zu wollen, der (sanktionsbewehrte) fachdisziplinäre Konsens und die Kohärenz neuer Erkenntnis mit den etablierten Begriffsrahmen und überkommenen Überzeugungssystemen lassen noch breiten Raum für weitere Forschungen. Hinzu kommt, dass sich gewiss auch weitere Restriktionen werden finden lassen, durch welche die hier genannten ergänzt werden müssen.

Konnte eine vollständige Theorie in diesem Kapitel nicht entwickelt werden, so konnten zumindest einige theoretische Voraussetzungen einer Vereinbarung von Standortgebundenheit und Objektivität vorgelegt werden. Über die philosophische Diskussion hinaus, die kaum noch weiterer Überzeugungsarbeit bedarf, wenn es um die Perspektivität objektiver Erkenntnis geht, kann über die Idee einer probeweisen Zusammenstellung von Sachverhalten in Propositionen und die interne Zuordnung von Begriffen zu Gegenständen und die damit verbundene Abhängigkeit von jeweils einschlägigen Begriffsrahmen der Propositionen der Weg beschritten werden, um Korrespondenz auch dann plausibel zu konzipieren, wenn vom po- 
sitivistischen Erkenntnismodell abgegangen wird. Der epistemisch-begriffliche Zugang zu den bestehenden Sachverhalten ist damit an den Standort des Erkenntnisobjekts gebunden, während dagegen die logisch-semantische Relation zwischen den begriffsrahmenabhängigen Propositionen und den durch sie ausgedrückten Sachverhalten die der Korrespondenz ist (wenn der ausgedrückte Sachverhalt besteht).

Ergänzend konnte der Einfluss der jeweiligen Elemente des Standortes zu diesen abstrakten Vorklärungen hinzugefügt werden, wobei auch hier eine ausgearbeitete Theorie historischer Standortgebundenheit die Lücke, die zwischen diesen allgemeinen philosophisch-semantischen Bedingungen und der geschichtsspezifischen Funktionsweise des Standortes klafft, schließen müsste. 
